\title{
The 482Ser of PPARGC1A and 12Pro of PPARG2 Alleles Are Associated with Reduction of Metabolic Risk Factors Even Obesity in a Mexican-Mestizo Population
}

\author{
Mónica Vázquez-Del Mercado, ${ }^{1,2,3}$ Milton-Omar Guzmán-Ornelas, ${ }^{1,2,4}$ \\ Fernanda-Isadora Corona Meraz, ${ }^{2,4}$ Clara-Patricia Ríos-Ibarra, ${ }^{5}$ \\ Eduardo-Alejandro Reyes-Serratos, ${ }^{5}$ Jorge Castro-Albarran, ${ }^{4,6,7}$ \\ Sandra-Luz Ruíz-Quezada, ${ }^{4,6}$ and Rosa-Elena Navarro-Hernández ${ }^{1,2,4,7}$ \\ ${ }^{1}$ Instituto de Investigación en Reumatología y del Sistema Musculo Esquelético, Centro Universitario de Ciencias de la Salud, \\ Universidad de Guadalajara, Sierra Mojada No. 950, Colonia Independencia, 44340 Guadalajara, JAL, Mexico \\ ${ }^{2}$ Centro Universitario de Ciencias de la Salud, Universidad de Guadalajara, Sierra Mojada No. 950, Colonia Independencia, \\ 44340 Guadalajara, JAL, Mexico \\ ${ }^{3}$ Servicio de Reumatología, Hospital Civil "Dr. Juan I. Menchaca”, Salvador Quevedo y Zubieta No. 750, Colonia Independencia, \\ 44340 Guadalajara, JAL, Mexico \\ ${ }^{4} U D G$-CA-701, Grupo de Investigación Inmunometabolismo en Enfermedades Emergentes (GIIEE), Centro Universitario de Ciencias \\ de la Salud, Universidad de Guadalajara, Sierra Mojada No. 950, Colonia Independencia, 44340 Guadalajara, JAL, Mexico \\ ${ }^{5}$ Tecnológico de Monterrey, Campus Guadalajara, Avenida General Ramón Corona No. 2514, Colonia Nuevo México, \\ 45201 Zapopan, JAL, Mexico \\ ${ }^{6}$ Departamento de Farmacobiología, Centro Universitario de Ciencias Exactas e Ingenierías, Universidad de Guadalajara, \\ Boulevard Marcelino García Barragán 1421, 44430 Guadalajara, JAL, Mexico \\ ${ }^{7}$ HMIELM, Secretaria de Salud Jalisco, Avenida Constituyentes 1075, Colonia Moderna, 44190 Guadalajara, JAL, Mexico
}

Correspondence should be addressed to Rosa-Elena Navarro-Hernández; rosa_elena_n@hotmail.com

Received 1 September 2014; Revised 19 November 2014; Accepted 23 November 2014

Academic Editor: Tiziano Verri

Copyright (C) 2015 Mónica Vázquez-Del Mercado et al. This is an open access article distributed under the Creative Commons Attribution License, which permits unrestricted use, distribution, and reproduction in any medium, provided the original work is properly cited.

\begin{abstract}
The aim of this study was to investigate the relationship between functional polymorphisms Gly482Ser in PPARGC1A and Pro12Ala in PPARG2 with the presence of obesity and metabolic risk factors. We included 375 individuals characterized as Mexican-Mestizos and classified by the body mass index (BMI). Body dimensions and distribution of body fat were measured. The HOMA-IR and adiposity indexes were calculated. Adipokines and metabolic profile quantification were performed by ELISA and routine methods. Genetic polymorphisms were determined by polymerase chain reaction restriction fragment length polymorphism analysis. A difference between obese and nonobese subjects in polymorphism PPARGC1A distribution was observed. Among obese individuals, carriers of genotype $482 \mathrm{Gly} / \mathrm{Gly}$ were observed to have decreased body fat, BMI, and body fat ratio versus 482 Ser/Ser carriers and increased resistin and leptin levels in carriers Gly+ phenotype versus Gly- phenotype. Subjects with PPARG2 Ala- phenotype (genotype 12Pro/Pro) showed a decreased HOMA-IR index versus individuals with Ala+ phenotype (genotypes 12Pro/Ala plus $12 \mathrm{Ala} / \mathrm{Ala})$. We propose that, in obese Mexican-Mestizos, the combination of alleles 482Ser in PPARGC1A and 12Pro in PPARG2 represents a reduced metabolic risk profile, even when the adiposity indexes are increased.
\end{abstract}

\section{Introduction}

Obesity is the result of a positive energetic balance that has been maintained for a long period of time. It is characterized by an excessive and continuous fat deposit in adipose tissue and is one of the most common metabolic disorders [1]. Obesity is considered a major factor that triggers metabolic risk and the development of secondary chronic illness [2]. 
The susceptibility of a subject to develop obesity will depend on different factors such as the repertoire of individual variations in an ensemble of relevant genes, their history of exposure to environmental risk factors, and the interaction between the lifestyle and metabolism, which is also modulated by the genetic components [3-5].

The identification of diverse molecular mechanisms related to energy metabolism has allowed the definition of strategies for searching genes implied in obesity. Among candidate genes that have been studied, PPARGC1A (peroxisome proliferator-activated receptor gamma, coactivator 1 alpha) and PPARG2 (peroxisome proliferator-activated receptor gamma 2) follow the same metabolic pathway $[1,2$, 6].

In human beings, PPARGC1A gene is located on chromosome 4p15.1 and codes for a 798 amino-acid protein (PGC$1 \alpha$ ), which is highly expressed on tissues where mitochondria are abundant and oxidative metabolism is activated, such as heart and skeletal muscle. It also plays a role in the pathogenesis of obesity by favoring the preadipocytes differentiation into adipocytes and by regulating the energy balance [7]. In PPARGC1A, a polymorphism that consists in a transition at codon 482 (rs8192678) was associated with chronic diseases [8-13].

The PPARG2 gene codes for PPAR $\gamma 2$ protein that is mainly expressed in adipose tissue and is the central engine in adipocyte differentiation. When activated by an agonist ligand in preadipocytes, a differentiation program is stimulated; in turn, morphological changes, lipid accumulation, and distinctive adipocyte gene expression take place [14-17].

PPARG2 is located on chromosome 3 and its polymorphism Prol2Ala, rs1801282 is located in exon B. In vitro analyses on its functionality have shown controversial results regarding the $12 \mathrm{Ala}$ allele and the reduction in the transcriptional activity of PPARG2, while the polymorphism's involvement in T2DM is reported in several studies [13, 1820].

In this context, it has been shown that obesity is due mainly to an increase in adiposity, where many functional genes are altered; among them are PPARG2 and its coactivator PPARGC1A. In adipose tissue, the expression of these genes is modified unfavorably reflecting altered adipogenesis and glucose metabolism. The Gly482Ser and Prol2Ala polymorphisms have been related to the development and severity of metabolic diseases, which suggests functional modification of the protein. Hence, the main objective of this study was to identify the relationship between polymorphisms Gly482Ser in PPARGC1A and Pro12Ala in PPARG2 and metabolic risk factors in normal, preobese, and obese Western MexicanMestizo population.

\section{Materials and Methods}

2.1. Study Design. A cross sectional study was performed. Each selected subject was requested to sign an informed written consent approved by the IRB committee of Centro Universitario de Ciencias de la Salud, Universidad de Guadalajara. A medical doctor confirmed a clinical healthy condition and stable weight for the past three months in every subject. Individuals that presented acute or chronic diseases, infections, and T2DM were not included.

The selected subjects were older than 18 years old, Mexican-Mestizos of Western of Mexico [21]. They were classified based on two criteria as follows: (1) normal (BMI: $18.5-24.9 \mathrm{~kg} / \mathrm{m}^{2}$ ), preobese (BMI: $25-29.9 \mathrm{~kg} / \mathrm{m}^{2}$ ), and obese $\left(\mathrm{BMI}>30.0 \mathrm{~kg} / \mathrm{m}^{2}\right)$ and (2) with obesity $\left(\mathrm{BMI}>30.0 \mathrm{~kg} / \mathrm{m}^{2}\right)$ and without obesity (BMI: $18.5-29.9 \mathrm{~kg} / \mathrm{m}^{2}$ ) [22].

2.2. Data Collection Methods. The study group constituted of 375 individuals. The clinical and nutritional evaluation data were obtained through questionnaires and general physical examinations.

\subsection{Body Dimensions and Distribution of Body Fat Storage} Measurements Included. Height (Seca GmbH \& Co KG. Hamburg, Germany, stadiometer to accurateness of $1.0 \mathrm{~mm}$ ), body weight, and body composition (measured with the electric bioimpedance method to the precision of $0.1 \mathrm{~kg}$ by TANITA's TBF304 system; Tokyo, Japan) [23, 24], waist and hip circumferences [25] with GULICK fiberglass retractable measurement tape (North Coast Medical Inc., Gilroy, CA). The obesity and adiposity indexes were calculated according to anthropometric indicators measurement guides: body mass index $\left(\mathrm{kg} / \mathrm{m}^{2}\right)=$ weight $(\mathrm{kg}) /$ height $^{2}(\mathrm{~m})$ [26]; body fat ratio $=$ total body fat mass $(\mathrm{kg}) /$ height $^{2}(\mathrm{~m})$ [27]; waist hip/ratio $(\mathrm{WHR})=$ waist circumference $(\mathrm{WC}, \mathrm{cm}) /$ hip circumference $(\mathrm{cm})$ [28]; waist height ratio $(\mathrm{WthR})=$ waist circumference $(\mathrm{cm}) /$ height $(\mathrm{cm})$ [29]; conicity index $=$ WC $(\mathrm{cm}) / 0.109 \sqrt{ }($ weight $(\mathrm{kg}) /$ height $(\mathrm{cm}))$ [30]; abdominal volume index $(L)=\left(\mathrm{WC}^{2}+(0.7 \mathrm{~cm})(\mathrm{WC}-\mathrm{HC})^{2}\right) / 1000$ [28]; total adipose area $\left(\mathrm{cm}^{2}\right)=\mathrm{WC}^{2} / 4 \pi$ [29]. The homeostasis model assessment-insulin resistance (HOMA-IR) [31] index was calculated.

\subsection{Adipokines and Metabolic Markers Profile Quantification.} Enzymatic and immunoturbidimetry assays (Randox Laboratories; 55 Diamond Road, Crumlin Co. Antrim, Northern Ireland, UK) were performed to quantify basal serum glucose, and lipid profile (i.e., triglycerides, total cholesterol, high and low density lipoproteins cholesterol (HDLc and LDLc, resp.), apolipoprotein A-1, and apolipoprotein B). Very low density lipoprotein cholesterol (VLDLc) was quantified using the Friedewald formula [32].

ELISA was used to quantify basal serum insulin levels (detection limit: $0.399 \mu \mathrm{IU} / \mathrm{mL}$ ), adiponectin (detection limit: $0.019 \mathrm{ng} / \mathrm{mL}$ ), leptin (detection limit: $0.42 \mathrm{ng} / \mathrm{mL}$ ); (ALPCO Diagnostics 26-G Keewaydin Drive, Salem NH 03079) and resistin levels (detection limit: $0.4 \mathrm{ng} / \mathrm{mL}$; Enzo Life Sciences, Inc.; New York, NY, USA).

2.5. Genotyping Techniques. To identify genetic polymorphisms, genomic DNA was isolated from total blood by the modified Miller's method [26] and stored at $-20^{\circ} \mathrm{C}$ until genotyping. 
The collected DNA underwent polymerase chain reaction- (PCR-) restriction fragment length polymorphism (RFLP) analysis; primers were PPARGC1A polymorphism F: $5^{\prime}$-TGCTACCTGAGAGAGACTTTG-3', R: 5'-CTTTCATCTTCGCTGTCATC-3', PPARG2: F: $5^{\prime}$-CAGTGTGGCAATATTTTCCCTGTA- $3^{\prime}$, and R: 5'GTATCAGTGAAGGAATCGCTTTCC- $3^{\prime}$ (this primer containing one nucleotide mismatch (underlined), which made it possible to use the restriction enzyme $M s p I$ ).

The PCR was performed with a $25 \mu \mathrm{L}$ reaction mixture (100 ng of DNA, $2 \mathrm{nM}$ of each primer, $2.5 \mathrm{mM}$ of each $\mathrm{dNTP}, 1.5 \mathrm{mM}$ of $\mathrm{MgCl}_{2}, 0.25 \mathrm{U}$ Taq polymerase, and $1 \mathrm{X}$ PCR buffer, (Invitrogen Life Technologies; Thermo Fisher Scientific Inc.)). PCR was performed with an initial melting step of $4 \mathrm{~min}$ at $94^{\circ} \mathrm{C}$, followed by 35 cycles of $30 \mathrm{~s}$ at $94^{\circ} \mathrm{C}, 30 \mathrm{~s}$ at $56 / 60^{\circ} \mathrm{C}$ (Gly482Ser and Pro12Ala polymorphisms, resp.), $30 \mathrm{~s}$ at $72^{\circ} \mathrm{C}$, and a final elongation step of $4 \mathrm{~min}$ at $72^{\circ} \mathrm{C}$.

To determine the changes in specific alleles of each individual, PCR products were submitted to a digestive process using the MspI enzyme (New England BioLabs Inc.; 240 Country Road, Ipswich, MA).

The obtained restriction fragments (149 and $111 \mathrm{bp}$ for Gly allele, and $260 \mathrm{bp}$ for Ser allele,/326 and $25 \mathrm{bp}$ for Pro allele, and $351 \mathrm{bp}$ for Ala allele) were observed in $4 \%$ agarose gels stained with ethidium bromide and photographed under UV light. To ensure the accuracy of genotype data, we used internal controls and repetitive experiments; that is, all samples were repeated at random to verify the reproducibility, with positive controls in each experiment. The genotyping success rate was $100 \%$.

2.6. Statistical Analysis. The statistical analysis was made with the software SPSS v21 (IBM Inc., Chicago, IL, USA) and GraphPad Prism v6.01 (@2014 Inc. 2236, Avenida de la Playa, La Jolla, CA 92037). Results are given as mean \pm SD or percentages.

The clinical and laboratory characteristics of the study group were performed with the unpaired Student's $t$-test, and to compare quantitative data in the three groups studied (normal, preobese and obese), a one-way ANOVA and post hoc Tukey's test were used. Data from serum concentrations of metabolic biomarkers with body adipose tissue variables were subjected to Pearson or Spearman correlation tests.

The test for Hardy-Weinberg equilibrium [33] for individual loci was performed for deviation. Contingency tables $(2 \times 2,3 \times 2$ and $3 \times 3)$ with $\chi^{2}$ trend test or Fisher exact test, depending on the case, were used for testing the differences of genotype distributions and allele frequencies between all subgroups. Two genetic models were used for these analyses: (i) the dominant model where each SNP was modeled categorically and separated into three categories, one for each genotype, and (ii) the phenotype model, where each polymorphism was modeled into two categories, with two genotypes combined into one category, choosing one genotype as the reference group, (i.e., polymorphism PPARGC1A: phenotype Ser+ (Genotypes 482Ser/Ser plus 482Gly/Ser) and, phenotype Ser- (genotype 482Gly/Gly); polymorphism
PPARG2: phenotype A- (genotype 12Pro/Pro) and phenotype Ala+ (genotypes 12Pro/Ala plus 12Ala/Ala)).

Genotype and phenotype intergroup comparisons by means of all variables were performed using one-way ANOVA with Tukey's test and, Student $t$-test for normally distributed traits, analysis of ranks for traits with nonnormal distributions with Kruskal-Wallis and Mann-Whitney $U$ test, as appropriate. A two-tailed $P$-value less than 0.05 was considered statistically significant.

\section{Results}

3.1. Clinical and Demographic Characteristics of the Whole Group. The cohort was composed of 375 Mexican-Mestizos $(\bar{x}=37.8 \pm 13.5$ years old $)$ of which 133 were men $(\bar{x}=$ $36.2 \pm 13.3$ years old $)$ and 242 were women $(\bar{x}=38.6 \pm$ 13.6 years old). Twenty-one percent of obesity and $59 \%$ of overweight frequencies were noted on the whole group. Abdominal obesity frequencies were 3.2, 32.9, and $74.7 \%$ with dyslipidemias of $16.8,32.9$, and $31.3 \%$ in normal range, preobese and obese individuals, respectively. The adiposity measurements and metabolic markers profile according to BMI and correlations in the whole group studied are shown in Tables 1 and 2. A positive correlation of glucose and insulin levels, HOMA-IR, and lipid profile was seen along accumulation of abdominal fat and an increase in adiposity indexes, except HDLc and apolipoprotein A-1. These two lipoproteins correlated negatively with abdominal obesity indicators (Table 2).

3.2. Distribution of Polymorphisms of PPARGC1A and PPARG2 in the Whole Group. In our cohort, the observed and expected frequencies of polymorphisms seen in individuals with BMI in normal range are according with the HardyWeinberg principle.

When we analyzed the Gly482Ser polymorphism of PPARGC1A we observed a similar distribution of the genotypes in individuals classified according to the WHO criteria on BMI.

The analysis of polymorphism of PPARG2 showed a difference in genotypic distribution in individuals classified according to the WHO criteria on BMI. We observed a difference in genotype and phenotype distribution in subjects without obesity when compared to individuals designated as obese (Table 3).

\subsection{Body Distribution and Clinical Measurements in Normal,} Preobese and Obese BMI Subjects by Genotypes and Phenotypes of PPARGC1A and PPARG2. Body fat distribution characteristics of the group are shown according to genotypes and phenotypes in normal range (Table 4(a)), preobese (Table 4(b)) and obese BMI subjects (Table 4(c)).

A difference in the level of glucose was found in the normal range subjects bearing genotype Gly/Ser versus Ser/Ser, while a difference in triglycerides level was observed in the preobese subjects when comparing Ser- versus Ser+ phenotypes, and in HDLc levels for Ala- versus Ala+ phenotypes (Table 4(b)). 
TABLE 1: Body dimensions, distribution of body fat storage, and metabolic markers profile according to BMI.

\begin{tabular}{|c|c|c|c|}
\hline Classification & Normal range & Preobese & Obese \\
\hline BMI $\left(\mathrm{kg} / \mathrm{m}^{2}\right)$ & [22.4 (18.5-24.9)] & [27.4 (25.1-29.6)] & {$[34.1(30.3-45.4)]$} \\
\hline$n$ & 153 & 144 & 78 \\
\hline \multicolumn{4}{|l|}{ Measurements } \\
\hline Height $(\mathrm{cm})^{\&}$ & $164.7 \pm 0.08$ & $163.5 \pm 0.09$ & $163.4 \pm 0.09$ \\
\hline Body weight $(\mathrm{kg})^{*}$ & $61.2 \pm 8.6$ & $73.5 \pm 9.3$ & $91.4 \pm 13.9$ \\
\hline Total body fat mass $(\mathrm{kg})^{*}$ & $14.4 \pm 5.7$ & $22.9 \pm 5.2$ & $36.2 \pm 8.9$ \\
\hline Body fat ratio* & $5.34 \pm 2.18$ & $8.56 \pm 2.05$ & $13.61 \pm 3.38$ \\
\hline Waist circumference $(\mathrm{cm})^{*}$ & $78.3 \pm 8.2$ & $91.6 \pm 8.1$ & $106.5 \pm 10.8$ \\
\hline Hip circumference $(\mathrm{cm})^{*}$ & $95.7 \pm 6.1$ & $102.6 \pm 5.2$ & $116.9 \pm 10.2$ \\
\hline $\mathrm{WHR}^{+}$ & $0.85 \pm 0.12$ & $0.86 \pm 0.12$ & $0.90 \pm 0.10$ \\
\hline WthR ${ }^{*}$ & $0.47 \pm 0.04$ & $0.56 \pm 0.04$ & $0.64 \pm 0.06$ \\
\hline Total adipose area $\left(\mathrm{cm}^{2}\right)^{*}$ & $493 \pm 105$ & $672 \pm 119$ & $896 \pm 183$ \\
\hline Conicity index ${ }^{*}$ & $1.18 \pm 0.10$ & $1.25 \pm 0.08$ & $1.29 \pm 0.09$ \\
\hline Abdominal volume index $(\mathrm{L})^{*}$ & $12.65 \pm 2.54$ & $17.04 \pm 2.90$ & $22.72 \pm 4.55$ \\
\hline Glucose $(\mathrm{mg} / \mathrm{dL})^{*}$ & $87 \pm 11$ & $96 \pm 12$ & $98 \pm 13$ \\
\hline Insulin $(\mu \mathrm{IU} / \mathrm{mL})^{*}$ & $14.1 \pm 24.3$ & $16.8 \pm 13.9$ & $25.1 \pm 29.9$ \\
\hline HOMA-IR* & $2.97 \pm 5.05$ & $4.00 \pm 3.41$ & $5.89 \pm 7.03$ \\
\hline Triglycerides $(\mathrm{mg} / \mathrm{dL})^{+}$ & $115 \pm 64$ & $167 \pm 103$ & $153 \pm 79$ \\
\hline Total cholesterol $(\mathrm{mg} / \mathrm{dL})^{+}$ & $172 \pm 37$ & $192 \pm 41$ & $192 \pm 31$ \\
\hline $\operatorname{HDLc}(\mathrm{mg} / \mathrm{dL})^{\&}$ & $40.7 \pm 16.0$ & $37.2 \pm 13.1$ & $37.9 \pm 14.2$ \\
\hline $\operatorname{LDLc}(\mathrm{mg} / \mathrm{dL})^{*}$ & $105 \pm 35$ & $113 \pm 36$ & $117 \pm 26$ \\
\hline $\operatorname{VLDLc}(\mathrm{mg} / \mathrm{dL})^{+}$ & $23.1 \pm 12.9$ & $33.0 \pm 20.5$ & $30.4 \pm 15.9$ \\
\hline Apolipoprotein A-1 (mg/dL) $)^{\&}$ & $118 \pm 29$ & $112 \pm 22$ & $117 \pm 27$ \\
\hline Apolipoprotein B (mg/dL) ${ }^{+}$ & $98 \pm 28$ & $124 \pm 34$ & $115 \pm 27$ \\
\hline Total sAdiponectin $(\mathrm{ng} / \mathrm{mL})^{+}$ & $7570 \pm 340$ & $3665 \pm 293$ & $4489 \pm 351$ \\
\hline sResistin $(\mathrm{ng} / \mathrm{mL})^{+}$ & $8.4 \pm 2.5$ & $9.7 \pm 3.2$ & $10.5 \pm 3.7$ \\
\hline sLeptin $(\mathrm{ng} / \mathrm{mL})^{*}$ & $17.6 \pm 14.6$ & $23.8 \pm 15.4$ & $57.5 \pm 30.7$ \\
\hline Abdominal obesity (\%) & 3.2 & 32.9 & 74.7 \\
\hline Dyslipidemia (\%) & 16.8 & 32.9 & 31.3 \\
\hline
\end{tabular}

Data are means \pm SD. One-way ANOVA test and Tukey post hoc test of normal range versus preobesity and obesity, and preobesity versus obesity, ${ }^{*}$ all measurements were different $(P<0.05),{ }^{+}$exception where only normal range versus obesity was different. ${ }^{8}$ No signifies differences were found. BMI: body mass index; WHR: waist-hip ratio; WthR: waist height ratio; HOMA-IR: homeostasis model assessment-insulin resistance; HDLc, LDLc, and VLDLc: high, low, and very low density lipoprotein cholesterol, respectively.

On the other hand, in obese individuals, we observed an increase in metabolic markers (glucose, insulin, and LDLc), increased HOMA-IR index, and low levels of total adiponectin in Ala+ versus Ala- phenotype carriers.

In carriers Ser+ versus Ser- phenotypes, a decrease of triglycerides and apolipoprotein $\mathrm{B}$ and increase of apolipoprotein A-1 levels were observed. Body dimensions (body weight, total body fat mass, and hip circumference), adiposity indexes (BMI, body fat ratio, and WHR), and conicity index were increased in carriers of polymorphic alleles 482 Ser and 12Ala, respectively (Table $4(c)$ ).

3.4. Metabolic, Adiposity Markers, Adipokines in the Whole Group according to Genotypes and Phenotypes of PPARGC1A and PPARG2. When comparing subjects carrying polymorphic genotype $482 \mathrm{Ser} / \mathrm{Ser}$ versus the wild-type genotype (i.e.: $482 \mathrm{Gly} / \mathrm{Gly}$ ), a decrease in glucose and apolipoprotein B levels was observed (Figures 1(a) and 1(b)). In the carriers of
12Ala/Ala polymorphic genotype, increase in dimensions and abdominal fat accumulation was observed when compared to carriers of the wild type genotype 12Pro/Pro and heterozygotes 12Pro/Ala (Figures 1(c) and 1(d)).

The most relevant data were obtained in obese individuals (i.e.: $\mathrm{BMI}>30 \mathrm{~kg} / \mathrm{m}^{2}$ ).

(i) Increase in body weight, total body fat mass, hip circumference, BMI, and body fat ratio, was seen in subjects carrying genotype Ser/Ser in comparison to carriers of genotypes Gly/Gly and Gly/Ser (Figures 2(a), and 2(b), resp.), as well as a parallel decrease in apolipoprotein B levels and WHR in carriers of Ser/Ser genotype individuals (Figures 2(c) and 2(b), resp.).

(ii) Increase in LDLc levels in carriers of genotype $12 \mathrm{Ala} / \mathrm{Ala}$ was seen as compared to carriers of genotype 12 Pro/Pro. Also, an increase in the magnitude 
TABLE 2: Correlations of metabolic markers profile with body adiposity measurements.

\begin{tabular}{|c|c|c|c|c|c|c|c|c|c|c|}
\hline \multirow{2}{*}{ Measurements } & Glucose & Insulin & HOMA-IR & Triglycerides & Total cholesterol & HDLc & LDLc & VLDLc & Apo A-1 & Apo B \\
\hline & & \multicolumn{9}{|c|}{$\begin{array}{c}{ }^{+} \text {Correlation, } \\
P\end{array}$} \\
\hline \multirow{2}{*}{ Body weight (kg) } & $0.270^{* *}$ & $0.335^{* *}$ & $0.353^{* *}$ & $0.313^{* *}$ & $0.215^{* *}$ & -0.082 & $0.116^{*}$ & $0.308^{* *}$ & -0.113 & $0.346^{* *}$ \\
\hline & $<0.0001$ & $<0.0001$ & $<0.0001$ & $<0.0001$ & $<0.0001$ & 0.114 & 0.026 & $<0.0001$ & 0.175 & $<0.0001$ \\
\hline \multirow{2}{*}{ BMI $\left(\mathrm{kg} / \mathrm{m}^{2}\right)$} & $0.380^{* *}$ & $0.397^{* *}$ & $0.435^{* *}$ & $0.350^{* *}$ & $0.322^{* *}$ & -0.033 & $0.195^{* *}$ & $0.344^{* *}$ & -0.018 & $0.345^{* *}$ \\
\hline & $<0.0001$ & $<0.0001$ & $<0.0001$ & $<0.0001$ & $<0.0001$ & 0.526 & $<0.0001$ & $<0.0001$ & 0.826 & $<0.0001$ \\
\hline \multirow{2}{*}{$\begin{array}{l}\text { Total body fat mass } \\
(\mathrm{kg})\end{array}$} & $0.339^{* *}$ & $0.324^{* *}$ & $0.366^{* *}$ & $0.284^{* *}$ & $0.284^{* *}$ & 0.058 & $0.173^{* *}$ & $0.284^{* *}$ & 0.026 & $0.317^{* *}$ \\
\hline & $<0.0001$ & $<0.0001$ & $<0.0001$ & $<0.0001$ & $<0.0001$ & 0.322 & 0.003 & $<0.0001$ & 0.834 & 0.006 \\
\hline \multirow{2}{*}{ Body fat ratio } & $0.362^{* *}$ & $0.409^{* *}$ & $0.443^{* *}$ & $0.336^{* *}$ & $0.309^{* *}$ & -0.028 & $0.191^{* *}$ & $0.330^{* *}$ & -0.021 & $0.343^{* *}$ \\
\hline & $<0.0001$ & $<0.0001$ & $<0.0001$ & $<0.0001$ & $<0.0001$ & 0.590 & $<0.0001$ & $<0.0001$ & 0.801 & $<0.0001$ \\
\hline \multirow{2}{*}{$\begin{array}{l}\text { Waist circumference } \\
(\mathrm{cm})\end{array}$} & $0.325^{* *}$ & $0.271^{* *}$ & $0.305^{* *}$ & $0.401^{* *}$ & $0.375^{* *}$ & $-0.124^{*}$ & $0.268^{* *}$ & $0.396^{* *}$ & $-0.182^{*}$ & $0.467^{* *}$ \\
\hline & $<0.0001$ & $<0.0001$ & $<0.0001$ & $<0.0001$ & $<0.0001$ & 0.026 & $<0.0001$ & $<0.0001$ & 0.041 & $<0.0001$ \\
\hline \multirow{2}{*}{$\begin{array}{l}\text { Hip circumference } \\
(\mathrm{cm})\end{array}$} & $0.224^{* *}$ & $0.293^{* *}$ & $0.305^{* *}$ & $0.177^{* *}$ & $0.243^{* *}$ & 0.042 & $0.146^{* *}$ & $0.165^{* *}$ & 0.011 & $0.200^{*}$ \\
\hline & $<0.0001$ & $<0.0001$ & $<0.0001$ & 0.001 & $<0.0001$ & 0.455 & 0.009 & 0.003 & 0.903 & 0.021 \\
\hline \multirow{2}{*}{ WHR } & $0.325^{* *}$ & $0.271^{* *}$ & $0.305^{* *}$ & $0.401^{* *}$ & $0.375^{* *}$ & $-0.124^{*}$ & $0.268^{* *}$ & $0.396^{* *}$ & $-0.182^{*}$ & $0.467^{* *}$ \\
\hline & $<0.0001$ & $<0.0001$ & $<0.0001$ & $<0.0001$ & $<0.0001$ & 0.026 & $<0.0001$ & $<0.0001$ & 0.041 & $<0.0001$ \\
\hline \multirow{2}{*}{ Conicity index } & $0.259^{* *}$ & 0.092 & $0.128^{*}$ & $0.368^{* *}$ & $0.360^{* *}$ & $-0.156^{* *}$ & $0.281^{* *}$ & $0.366^{* *}$ & $-0.256^{* *}$ & $0.462^{* *}$ \\
\hline & $<0.0001$ & 0.099 & 0.022 & $<0.0001$ & $<0.0001$ & 0.005 & $<0.0001$ & $<0.0001$ & 0.004 & $<0.0001$ \\
\hline \multirow{2}{*}{$\begin{array}{l}\text { Total adipose area } \\
\left(\mathrm{cm}^{2}\right)\end{array}$} & $0.325^{* *}$ & $0.271^{* *}$ & $0.305^{* *}$ & $0.401^{* *}$ & $0.375^{* *}$ & $-0.124^{*}$ & $0.268^{* *}$ & $0.396^{* *}$ & $-0.182^{*}$ & $0.467^{* *}$ \\
\hline & $<0.0001$ & $<0.0001$ & $<0.0001$ & $<0.0001$ & $<0.0001$ & 0.026 & $<0.0001$ & $<0.0001$ & 0.041 & $<0.0001$ \\
\hline \multirow{2}{*}{$\begin{array}{l}\text { Abdominal volume } \\
\text { index (L) }\end{array}$} & $0.324^{* *}$ & $0.275^{* *}$ & $0.308^{* *}$ & $0.394^{* *}$ & $0.373^{* *}$ & $-0.117^{*}$ & $0.266^{* *}$ & $0.390^{* *}$ & $-0.176^{*}$ & $0.463^{* *}$ \\
\hline & $<0.0001$ & $<0.0001$ & $<0.0001$ & $<0.0001$ & $<0.0001$ & 0.035 & $<0.0001$ & $<0.0001$ & 0.049 & $<0.0001$ \\
\hline
\end{tabular}

HOMA-IR: homeostasis model assessment-insulin resistance; Glucose, HDLc, LDLc, and VLDLc: high, low, and very low density lipoprotein cholesterol, respectively; Apo: apolipoprotein $\mathrm{mg} / \mathrm{dL}$. Insulin, $\mu \mathrm{IU} / \mathrm{mL} .{ }^{* *}$ Significant correlation at 0.01 level. ${ }^{*}$ Significant correlation at 0.05 level. ${ }^{+} r$ Pearson/Rho of Spearman correlations test.

TABLE 3: Distribution of Gly482Ser in PPARGC1A and Pro12Ala in PPARG2 polymorphisms in Mexican-Mestizo population.

\begin{tabular}{|c|c|c|c|c|c|c|c|}
\hline \multirow{2}{*}{$\begin{array}{l}\text { Study group } \\
P P A R G C 1 A \text { Gly482Ser } \\
\text { rs8192678 }\end{array}$} & \multicolumn{3}{|c|}{ Genotype $n(\%)$} & \multicolumn{2}{|c|}{ Phenotype $n(\%)$} & \multicolumn{2}{|c|}{ Allele $n(\%)$} \\
\hline & Gly/Gly (Ser-) & Gly/Ser & Ser/Ser (Gly-) & Gly+ & Ser+ & Gly & Ser \\
\hline Normal range & $77(50)$ & $60(39)$ & $16(11)$ & $137(89)$ & $76(50)$ & $214(70)$ & $92(30)$ \\
\hline Preobese & $74(51)$ & $54(38)$ & $16(11)$ & $128(89)$ & $70(49)$ & $202(70)$ & $86(30)$ \\
\hline Obese & $38(49)$ & $31(40)$ & $9(11)$ & $69(89)$ & $40(51)$ & $107(70)$ & $49(30)$ \\
\hline$P$ & & ${ }^{1} \mathrm{NS}$ & & ${ }^{2} \mathrm{NS}$ & ${ }^{2} \mathrm{NS}$ & \multicolumn{2}{|c|}{${ }^{5} \mathrm{NS}$} \\
\hline Without obesity & $151(51)$ & $114(38)$ & $32(11)$ & $265(89)$ & $146(49)$ & $416(70)$ & $178(30)$ \\
\hline$P$ & & ${ }^{3} \mathrm{NS}$ & & ${ }^{4} \mathrm{NS}$ & ${ }^{4} \mathrm{NS}$ & \multicolumn{2}{|c|}{${ }^{6} \mathrm{NS}$} \\
\hline $\begin{array}{l}\text { PPARG2 Pro12Ala } \\
\text { rs1801282 }\end{array}$ & Pro/Pro (Ala-) & Pro/Ala & Ala/Ala (Pro-) & Pro+ & Ala+ & Pro & Ala \\
\hline Normal range & $117(76)$ & $34(23)$ & $2(1)$ & $151(99)$ & $36(24)$ & $268(87)$ & $38(13)$ \\
\hline Preobese & $117(81)$ & $27(19)$ & $0(0)$ & $144(100)$ & $27(19)$ & $261(90)$ & $27(10)$ \\
\hline Obese & $61(78)$ & $13(17)$ & $4(5)$ & $74(95)$ & $17(22)$ & $135(87)$ & $21(13)$ \\
\hline$P$ & & ${ }^{1} \mathbf{0 . 0 4 7}$ & & ${ }^{2} 0.014$ & ${ }^{2} \mathrm{NS}$ & \multicolumn{2}{|c|}{${ }^{5} \mathrm{NS}$} \\
\hline Without obesity & $234(78)$ & $61(21)$ & $2(1)$ & $295(99)$ & $63(22)$ & $529(89)$ & $65(11)$ \\
\hline$P$ & & ${ }^{3} 0.017$ & & ${ }^{4} 0.019$ & ${ }^{4} \mathrm{NS}$ & \multicolumn{2}{|c|}{${ }^{6} \mathrm{NS}$} \\
\hline
\end{tabular}


TABLE 4: Body dimensions, distribution of body fat storage, and metabolic markers profile according to genotypes and phenotype.

(a) Normal range BMI subjects

\begin{tabular}{|c|c|c|c|c|c|c|c|c|}
\hline \multirow{3}{*}{ Measurements } & \multicolumn{4}{|c|}{ Polymorphism PPARGC1A } & \multicolumn{4}{|c|}{ Polymorphism PPARG2 } \\
\hline & & Genotypes & & Phenotype & & Genotypes & & Phenotype \\
\hline & Gly/Gly & Gly/Ser & Ser/Ser & Ser+ & Pro/Pro & Pro/Ala & Ala/Ala & $\mathrm{Ala}+$ \\
\hline$n$ & 77 & 60 & 16 & 76 & 117 & 34 & 2 & 36 \\
\hline Height $(\mathrm{cm})$ & $164.6 \pm 8.3$ & $164.7 \pm 9.3$ & $165.5 \pm 10.5$ & $164.9 \pm 9.5$ & $163.9 \pm 9.0$ & $167.5 \pm 7.8$ & $165.0 \pm 11.3$ & $167.3 \pm 7.8$ \\
\hline Body weight (kg) & $61.0 \pm 7.7$ & $60.9 \pm 9.5$ & $62.9 \pm 9.2$ & $61.4 \pm 9.4$ & $60.7 \pm 8.9$ & $62.9 \pm 6.8$ & $59.5 \pm 17.2$ & $62.7 \pm 7.2$ \\
\hline BMI $\left(\mathrm{kg} / \mathrm{m}^{2}\right)$ & $22.49 \pm 1.7$ & $22.32 \pm 1.6$ & $22.77 \pm 1.6$ & $22.4 \pm 1.6$ & $22.48 \pm 1.6$ & $22.40 \pm 1.6$ & $21.55 \pm 3.3$ & $22.3 \pm 1.7$ \\
\hline $\begin{array}{l}\text { Total body fat mass } \\
(\mathrm{kg})\end{array}$ & $14.1 \pm 5.2$ & $15.0 \pm 6.5$ & $13.8 \pm 5.8$ & $14.7 \pm 6.3$ & $14.6 \pm 5.8$ & $13.9 \pm 5.3$ & $15.6 \pm 11.7$ & $14.0 \pm 5.6$ \\
\hline Body fat ratio & $5.23 \pm 1.93$ & $5.60 \pm 2.48$ & $4.88 \pm 2.25$ & $5.46 \pm 2.43$ & $5.46 \pm 2.21$ & $4.95 \pm 2.08$ & $5.48 \pm 3.56$ & $4.98 \pm 2.11$ \\
\hline $\begin{array}{l}\text { Waist circumference } \\
(\mathrm{cm})\end{array}$ & $78.1 \pm 8.1$ & $78.1 \pm 8.4$ & $80.1 \pm 7.9$ & $78.5 \pm 8.2$ & $77.7 \pm 8.1$ & $80.4 \pm 7.9$ & $70.4 \pm 6.8$ & $80.1 \pm 8.0$ \\
\hline $\begin{array}{l}\text { Hip circumference } \\
(\mathrm{cm})\end{array}$ & $95.5 \pm 4.8$ & $95.9 \pm 7.8$ & $95.3 \pm 4.5$ & $95.8 \pm 7.1$ & $95.8 \pm 6.5$ & $95.4 \pm 4.5$ & $87.7 \pm 5.3$ & $95.2 \pm 4.6$ \\
\hline WHR & $0.82 \pm 0.08$ & $0.80 \pm 0.14$ & $0.84 \pm 0.07$ & $0.81 \pm 0.13$ & $0.80 \pm 0.11$ & $0.84 \pm 0.08$ & $0.79 \pm 0.02$ & $0.84 \pm 0.09$ \\
\hline WthR & $0.47 \pm 0.04$ & $0.47 \pm 0.05$ & $0.48 \pm 0.04$ & $0.48 \pm 0.05$ & $0.48 \pm 0.05$ & $0.48 \pm 0.05$ & $0.45 \pm 0.05$ & $0.48 \pm 0.05$ \\
\hline $\begin{array}{l}\text { Total adipose area } \\
\left(\mathrm{cm}^{2}\right)\end{array}$ & $490 \pm 104$ & $490 \pm 111$ & $515 \pm 97$ & $496 \pm 108$ & $486 \pm 105$ & $519 \pm 104$ & $394 \pm 92$ & $515 \pm 105$ \\
\hline Conicity index & $1.17 \pm 0.09$ & $1.18 \pm 0.12$ & $1.19 \pm 0.08$ & $1.19 \pm 0.12$ & $1.18 \pm 0.11$ & $1.20 \pm 0.11$ & $1.18 \pm 0.01$ & $1.20 \pm 0.11$ \\
\hline $\begin{array}{l}\text { Abdominal volume } \\
\text { index }(\mathrm{L})\end{array}$ & $12.5 \pm 2.49$ & $12.6 \pm 2.71$ & $13.1 \pm 2.33$ & $12.7 \pm 2.62$ & $12.5 \pm 2.5$ & $13.3 \pm 2.5$ & $10.1 \pm 0.02$ & $13.1 \pm 2.54$ \\
\hline Glucose $(\mathbf{m g} / \mathbf{d L})^{*}$ & $87 \pm 9.3$ & $89 \pm 12.1$ & $80 \pm 13.4$ & $87 \pm 12.7$ & $87 \pm 11.8$ & $85 \pm 8.6$ & $79 \pm 4.24$ & $85 \pm 8.5$ \\
\hline Insulin $(\mu \mathrm{IU} / \mathrm{mL})$ & $11.3 \pm 12.9$ & $18.4 \pm 35.5$ & $10.9 \pm 7.5$ & $16.9 \pm 31.8$ & $14.9 \pm 27.3$ & $11.4 \pm 9.4$ & $10.2 \pm 4.1$ & $11.3 \pm 9.2$ \\
\hline HOMA-IR & $2.40 \pm 2.89$ & $3.95 \pm 7.22$ & $1.89 \pm 1.11$ & $3.54 \pm 6.51$ & $3.51 \pm 5.67$ & $2.40 \pm 2.00$ & $1.98 \pm 0.70$ & $2.20 \pm 1.90$ \\
\hline Triglycerides (mg/dL) & $117 \pm 70$ & $113 \pm 57$ & $114 \pm 66$ & $113 \pm 58$ & $115 \pm 68$ & $118 \pm 53$ & $74 \pm 6$ & $115 \pm 53$ \\
\hline $\begin{array}{l}\text { Total cholesterol } \\
(\mathrm{mg} / \mathrm{dL})\end{array}$ & $172 \pm 32$ & $173 \pm 43$ & $171 \pm 39$ & $172 \pm 42$ & $172 \pm 36$ & $173 \pm 42$ & $159 \pm 60$ & $172 \pm 42$ \\
\hline HDLc (mg/dL) & $39 \pm 17.6$ & $41 \pm 16.1$ & $41 \pm 17.3$ & $41 \pm 16.2$ & $41 \pm 17.0$ & $38 \pm 16.9$ & $34 \pm 5.7$ & $16 \pm 2.8$ \\
\hline LDLc (mg/dL) & $106 \pm 29.4$ & $105 \pm 41.6$ & $105 \pm 42.2$ & $105 \pm 41.5$ & $104 \pm 35.3$ & $107 \pm 37.7$ & $110 \pm 53.9$ & $37 \pm 6.3$ \\
\hline VLDLc (mg/dL) & $23.5 \pm 14.1$ & $22.7 \pm 11.4$ & $22.9 \pm 13.4$ & $22.7 \pm 11.8$ & $23.1 \pm 13.6$ & $23.6 \pm 10.7$ & $14.9 \pm 1.3$ & $23.2 \pm 10.6$ \\
\hline $\begin{array}{l}\text { Apolipoprotein A-1 } \\
(\mathrm{mg} / \mathrm{dL})\end{array}$ & $118 \pm 24.4$ & $116 \pm 35.9$ & $125 \pm 20.3$ & $118 \pm 24.4$ & $117 \pm 30.9$ & $123 \pm 21.7$ & $112 \pm 11.7$ & $123 \pm 21.7$ \\
\hline $\begin{array}{l}\text { Apolipoprotein B } \\
(\mathrm{mg} / \mathrm{dL})\end{array}$ & $102 \pm 30.1$ & $97 \pm 28.9$ & $89 \pm 20.9$ & $102 \pm 30.1$ & $99 \pm 29.0$ & $96 \pm 27.4$ & $98 \pm 12.8$ & $97 \pm 10.3$ \\
\hline $\begin{array}{l}\text { Total sAdiponectin } \\
\text { (ng/mL) }\end{array}$ & $8119 \pm 4358$ & $6540 \pm 1808$ & $10361 \pm 4378$ & $8119 \pm 4358$ & $7756 \pm 3073$ & $7130 \pm 5673$ & $5046 \pm 2326$ & $5001 \pm 2236$ \\
\hline sResistin $(\mathrm{ng} / \mathrm{mL})^{\&}$ & $8.1 \pm 2.5$ & $8.4 \pm 2.3$ & $9.7 \pm 3.8$ & $8.1 \pm 2.5$ & $7.9 \pm 2.2$ & $11.2 \pm 1.5$ & $10.7 \pm 1.9$ & $11.3 \pm 1.5$ \\
\hline sLeptin (ng/mL) & $18.5 \pm 14.9$ & $18.0 \pm 15.1$ & $14.6 \pm 3.2$ & $18.4 \pm 14.9$ & $16.2 \pm 14.5$ & $20.8 \pm 17.4$ & $33.2 \pm 11.9$ & $23.9 \pm 15.5$ \\
\hline
\end{tabular}

$n=153$. Normal range BMI $18.5-24.9 \mathrm{~kg} / \mathrm{m}^{2}$. Data are means \pm SD. ${ }^{*}$ Gly $/$ Ser versus Ser $/$ Ser genotypes, $P=0.022 .{ }^{\text {\& }}$ ALA- versus ALA+ phenotypes $P=0.009$.

BMI: body mass index; WHR: waist-hip ratio; WthR: waist height ratio; HOMA-IR: Homeostasis model assessment-insulin resistance; HDLc, LDLc, and VLDLc: high, low, and very low density lipoprotein cholesterol, respectively.

(b) Preobese range BMI subjects

\begin{tabular}{|c|c|c|c|c|c|c|c|c|}
\hline \multirow{3}{*}{ Measurements } & \multicolumn{4}{|c|}{ Polymorphism PPARGC1A } & \multicolumn{4}{|c|}{ Polymorphism PPARG2 } \\
\hline & & Genotypes & & Phenotype & & Genotypes & & Phenotype \\
\hline & Gly/Gly & Gly/Ser & Ser/Ser & Ser+ & Pro/Pro & Pro/Ala & Ala/Ala & Ala+ \\
\hline$n$ & 74 & 54 & 16 & 70 & 117 & 27 & 0 & 27 \\
\hline Height $(\mathrm{cm})$ & $163.8 \pm 8.8$ & $164.8 \pm 9.77$ & $157.9 \pm 6.6$ & $163.2 \pm 9.6$ & $164.2 \pm 8.9$ & $160.5 \pm 9.8$ & - & $160.5 \pm 9.8$ \\
\hline Body weight (kg) & $74.0 \pm 9.3$ & $74.2 \pm 9.7$ & $68.5 \pm 5.7$ & $72.8 \pm 9.3$ & $74.0 \pm 8.9$ & $71.3 \pm 10.6$ & - & $71.3 \pm 10.6$ \\
\hline BMI $\left(\mathrm{kg} / \mathrm{m}^{2}\right)$ & $27.50 \pm 1.50$ & $27.20 \pm 1.32$ & $27.48 \pm 1.43$ & $27.27 \pm 1.34$ & $27.35 \pm 1.42$ & $27.55 \pm 1.45$ & - & $27.55 \pm 1.45$ \\
\hline $\begin{array}{l}\text { Total body fat mass } \\
(\mathrm{kg})\end{array}$ & $23.8 \pm 5.1$ & $22.4 \pm 5.5$ & $21.4 \pm 4.3$ & $22.1 \pm 5.2$ & $22.6 \pm 5.4$ & $23.8 \pm 4.4$ & - & $23.8 \pm 4.4$ \\
\hline
\end{tabular}


(b) Continued.

\begin{tabular}{|c|c|c|c|c|c|c|c|c|}
\hline \multirow{3}{*}{ Measurements } & \multicolumn{4}{|c|}{ Polymorphism PPARGC1A } & \multicolumn{4}{|c|}{ Polymorphism PPARG2 } \\
\hline & & Genotypes & & Phenotype & & Genotypes & & Phenotype \\
\hline & Gly/Gly & Gly/Ser & Ser/Ser & Ser+ & Pro/Pro & Pro/Ala & Ala/Ala & $\mathrm{Ala}+$ \\
\hline$n$ & 74 & 54 & 16 & 70 & 117 & 27 & 0 & 27 \\
\hline Body fat ratio & $8.78 \pm 2.04$ & $8.28 \pm 2.17$ & $8.61 \pm 1.78$ & $8.37 \pm 2.06$ & $8.40 \pm 2.16$ & $9.14 \pm 1.51$ & - & $9.14 \pm 1.51$ \\
\hline $\begin{array}{l}\text { Waist circumference } \\
(\mathrm{cm})\end{array}$ & $92.3 \pm 8.4$ & $91.2 \pm 8.2$ & $90.1 \pm 6.7$ & $91.0 \pm 7.8$ & $91.6 \pm 7.7$ & $91.5 \pm 9.8$ & - & $91.5 \pm 9.8$ \\
\hline $\begin{array}{l}\text { Hip circumference } \\
(\mathrm{cm})\end{array}$ & $103.6 \pm 5.1$ & $101.9 \pm 5.5$ & $100.8 \pm 3.8$ & $101.6 \pm 5.1$ & $102.6 \pm 5.3$ & $102.8 \pm 4.5$ & - & $102.8 \pm 4.5$ \\
\hline WHR & $0.90 \pm 0.14$ & $0.90 \pm 0.08$ & $0.90 \pm 0.07$ & $0.89 \pm 0.08$ & $0.89 \pm 0.12$ & $0.89 \pm 0.08$ & - & $0.89 \pm 0.08$ \\
\hline WthR & $0.56 \pm 0.04$ & $0.55 \pm 0.04$ & $0.57 \pm 0.03$ & $0.56 \pm 0.04$ & $0.56 \pm 0.04$ & $0.58 \pm 0.04$ & - & $0.58 \pm 0.04$ \\
\hline $\begin{array}{l}\text { Total adipose area } \\
\left(\mathrm{cm}^{2}\right)\end{array}$ & $682 \pm 125$ & $667 \pm 18$ & $649 \pm 95$ & $662 \pm 112$ & $672 \pm 112$ & $673 \pm 149$ & - & $673 \pm 149$ \\
\hline Conicity index & $1.26 \pm 0.08$ & $1.25 \pm 0.09$ & $1.26 \pm 0.07$ & $1.25 \pm 0.09$ & $1.25 \pm 0.08$ & $1.27 \pm 0.09$ & - & $1.27 \pm 0.09$ \\
\hline $\begin{array}{l}\text { Abdominal volume } \\
\text { index }(\mathrm{L})\end{array}$ & $17.30 \pm 3.08$ & $16.90 \pm 2.87$ & $16.43 \pm 2.29$ & $16.78 \pm 2.73$ & $17.04 \pm 2.74$ & $17.10 \pm 3.7$ & - & $17.07 \pm 3.70$ \\
\hline Glucose (mg/dL) & $97 \pm 12.1$ & $95 \pm 11.7$ & $92 \pm 13.5$ & $94 \pm 12.1$ & $95 \pm 11.3$ & $97 \pm 15.4$ & - & $97 \pm 15.4$ \\
\hline Insulin $(\mu \mathrm{IU} / \mathrm{mL})$ & $15.9 \pm 9.9$ & $18.9 \pm 19.1$ & $13.3 \pm 5.9$ & $17.6 \pm 17.1$ & $17.3 \pm 14.9$ & $14.5 \pm 7.5$ & - & $14.5 \pm 7.5$ \\
\hline HOMA-IR & $3.91 \pm 2.54$ & $4.43 \pm 4.63$ & $3.06 \pm 1.48$ & $4.11 \pm 4.15$ & $4.12 \pm 3.67$ & $3.52 \pm 1.99$ & - & $3.53 \pm 1.99$ \\
\hline $\begin{array}{l}\text { Triglycerides } \\
(\mathrm{mg} / \mathrm{dL})^{*}\end{array}$ & $186 \pm 124$ & $153 \pm 74$ & $133 \pm 60$ & $148 \pm 71$ & $173 \pm 108$ & $145 \pm 76$ & - & $145 \pm 76$ \\
\hline $\begin{array}{l}\text { Total cholesterol } \\
(\mathrm{mg} / \mathrm{dL})\end{array}$ & $195 \pm 43$ & $189 \pm 40$ & $188 \pm 31$ & $189 \pm 38$ & $193 \pm 44$ & $187 \pm 25$ & - & $187 \pm 25$ \\
\hline $\operatorname{HDLc}(\mathrm{mg} / \mathrm{dL})^{\&}$ & $37 \pm 13.6$ & $36 \pm 13.0$ & $32 \pm 12.2$ & $36 \pm 12.7$ & $36 \pm 12.7$ & $41 \pm 14.1$ & - & $41 \pm 14.1$ \\
\hline $\mathrm{LDLc}(\mathrm{mg} / \mathrm{dL})$ & $111 \pm 32.5$ & $115 \pm 41.3$ & $115 \pm 35.4$ & $115 \pm 39.7$ & $114 \pm 38.1$ & $107 \pm 25.6$ & - & $107 \pm 25.6$ \\
\hline $\operatorname{VLDLc}(\mathrm{mg} / \mathrm{dL})$ & $36.2 \pm 24.8$ & $30.5 \pm 14.7$ & $26.8 \pm 12.1$ & $29.6 \pm 14.2$ & $34.0 \pm 21.6$ & $29.1 \pm 15.3$ & - & $29.1 \pm 15.3$ \\
\hline $\begin{array}{l}\text { Apolipoprotein A-1 } \\
\text { (mg/dL) }\end{array}$ & $109 \pm 15.6$ & $120 \pm 31.5$ & $108 \pm 6.7$ & $118 \pm 29.1$ & $110 \pm 15.8$ & $124 \pm 37.5$ & - & $124 \pm 37.5$ \\
\hline $\begin{array}{l}\text { Apolipoprotein B } \\
\text { (mg/dL) }\end{array}$ & $129 \pm 40.5$ & $117 \pm 23.0$ & $113 \pm 9.0$ & $117 \pm 21.4$ & $125 \pm 35.4$ & $121 \pm 30.9$ & - & $121 \pm 30.9$ \\
\hline $\begin{array}{l}\text { Total sAdiponectin } \\
(\mathrm{ng} / \mathrm{mL})\end{array}$ & $3929 \pm 3145$ & $3563 \pm 2715$ & $3283 \pm 3107$ & $3485 \pm 2801$ & $3646 \pm 2745$ & $3721 \pm 3516$ & - & $3721 \pm 3516$ \\
\hline sResistin (ng/mL) & $8.9 \pm 2.6$ & $10.6 \pm 3.8$ & $10.5 \pm 4.9$ & $10.6 \pm 3.8$ & $9.3 \pm 3.4$ & $10.6 \pm 2.8$ & - & $10.6 \pm 2.8$ \\
\hline sLeptin (ng/mL) & $24.1 \pm 14.3$ & $20.2 \pm 14.9$ & $31.3 \pm 16.7$ & $23.6 \pm 16.1$ & $23.2 \pm 16.0$ & $26.0 \pm 13.5$ & - & $26.0 \pm 13.5$ \\
\hline
\end{tabular}

$n=144$. Preobese range, BMI $25.0-29.9 \mathrm{~kg} / \mathrm{m}^{2}$. Data are means \pm SD. ${ }^{*}$ Ser- versus Ser+ phenotypes, $P=0.030 .{ }^{\text {\& }}$ Ala - versus Ala + phenotypes, $P=0.039$.

(c) Obese range BMI subjects

\begin{tabular}{|c|c|c|c|c|c|c|c|c|}
\hline \multirow{3}{*}{ Measurements } & \multicolumn{4}{|c|}{ Polymorphism PPARGC1A } & \multicolumn{4}{|c|}{ Polymorphism PPARG2 } \\
\hline & & Genotypes & & Phenotype & & Genotypes & & Phenotype \\
\hline & Gly/Gly & Gly/Ser & Ser/Ser & Ser+ & Pro/Pro & Pro/Ala & Ala/Ala & Ala+ \\
\hline$n$ & 38 & 31 & 9 & 40 & 61 & 13 & 4 & 17 \\
\hline Height $(\mathrm{cm})$ & $162.9 \pm 9.3$ & $162.1 \pm 9.8$ & $170.5 \pm 1.3$ & $164.0 \pm 10.6$ & $164.2 \pm 9.9$ & $159.2 \pm 8.8$ & $166.8 \pm 13.4$ & $160.9 \pm 10.1$ \\
\hline Body weight $(\mathbf{k g})^{*}$ & $90.6 \pm 11.8$ & $87.4 \pm 13.4$ & $108.9 \pm 10.8$ & $92.2 \pm 15.6$ & $91.9 \pm 14.6$ & $89.7 \pm 10.9$ & $89.7 \pm 12.9$ & $89.7 \pm 11.0$ \\
\hline BMI $\left(\mathbf{k g} / \mathbf{m}^{2}\right)^{*}$ & $34.08 \pm 3.34$ & $33.18 \pm 0.55$ & $37.80 \pm 5.30$ & $34.21 \pm 4.38$ & $34.02 \pm 4.03$ & $35.41 \pm 3.47$ & $32.18 \pm 1.49$ & $34.64 \pm 3.38$ \\
\hline $\begin{array}{l}\text { Total body fat mass } \\
(\mathrm{kg})^{*}\end{array}$ & $34.4 \pm 7.8$ & $34.3 \pm 7.1$ & $47.7 \pm 9.2$ & $37.9 \pm 9.7$ & $36.7 \pm 8.8$ & $35.4 \pm 10.9$ & $32.4 \pm 4.5$ & $34.5 \pm 9.4$ \\
\hline Body fat ratio* & $13.12 \pm 3.23$ & $13.19 \pm 2.76$ & $16.48 \pm 0.42$ & $14.06 \pm 3.52$ & $13.73 \pm 3.32$ & $13.87 \pm 4.04$ & $11.81 \pm 2.35$ & $13.2 \pm 3.7$ \\
\hline $\begin{array}{l}\text { Waist circumference } \\
(\mathrm{cm})\end{array}$ & $106.4 \pm 11.7$ & $103.2 \pm 9.6$ & $110.9 \pm 9.6$ & $104.9 \pm 9.9$ & $105.7 \pm 10.9$ & $102.2 \pm 10.5$ & $113.1 \pm 7.9$ & $105.3 \pm 10.8$ \\
\hline $\begin{array}{l}\text { Hip circumference } \\
(\mathrm{cm})^{*}\end{array}$ & $115.8 \pm 9.6$ & $115.6 \pm 8.2$ & $127.6 \pm 14.3$ & $118.1 \pm 10.7$ & $117.5 \pm 10.3$ & $116.7 \pm 0.4$ & $110.0 \pm 5.9$ & $114.8 \pm 9.6$ \\
\hline $\mathrm{WHR}^{\circ \&}$ & $0.94 \pm 0.12$ & $0.89 \pm 0.08$ & $0.77 \pm 0.31$ & $0.87 \pm 0.16$ & $0.89 \pm 0.15$ & $0.90 \pm 0.11$ & $1.03 \pm 0.10$ & $0.93 \pm 0.12$ \\
\hline WthR & $0.65 \pm 0.07$ & $0.63 \pm 0.07$ & $0.65 \pm 0.08$ & $0.63 \pm 0.07$ & $0.64 \pm 0.07$ & $0.65 \pm 0.07$ & $0.68 \pm 0.04$ & $0.65 \pm 0.07$ \\
\hline
\end{tabular}


(c) Continued.

\begin{tabular}{|c|c|c|c|c|c|c|c|c|}
\hline \multirow{3}{*}{ Measurements } & \multicolumn{4}{|c|}{ Polymorphism PPARGC1A } & \multicolumn{4}{|c|}{ Polymorphism PPARG2 } \\
\hline & & Genotypes & & Phenotype & & Genotypes & & Phenotype \\
\hline & Gly/Gly & Gly/Ser & Ser/Ser & Ser+ & Pro/Pro & Pro/Ala & Ala/Ala & $\mathrm{Ala}+$ \\
\hline$n$ & 38 & 31 & 9 & 40 & 61 & 13 & 4 & 17 \\
\hline $\begin{array}{l}\text { Total adipose area } \\
\left(\mathrm{cm}^{2}\right)\end{array}$ & $911 \pm 198$ & $855 \pm 161$ & $984 \pm 167$ & $882 \pm 168$ & $898 \pm 186$ & $838 \pm 167$ & $1021 \pm 138$ & $891 \pm 176$ \\
\hline Conicity index ${ }^{\#}$ & $1.31 \pm 0.11$ & $1.28 \pm 0.09$ & $1.26 \pm 0.07$ & $1.28 \pm 0.08$ & $1.29 \pm 0.08$ & $1.25 \pm 0.14$ & $1.42 \pm 0.05$ & $1.30 \pm 0.15$ \\
\hline $\begin{array}{l}\text { Abdominal volume } \\
\text { index (L) }\end{array}$ & $23.08 \pm 4.92$ & $21.66 \pm 3.97$ & $24.98 \pm 4.25$ & $22.4 \pm 4.2$ & $22.8 \pm 4.6$ & $21.3 \pm 4.1$ & $25.7 \pm 3.5$ & $22.6 \pm 4.4$ \\
\hline Glucose $(\mathrm{mg} / \mathrm{dL})^{+}$ & $98 \pm 15.2$ & $97 \pm 11.4$ & $92 \pm 11.2$ & $96 \pm 11.4$ & $96 \pm 13.3$ & $104 \pm 13.3$ & $96 \pm 9.0$ & $103 \pm 13.8$ \\
\hline Insulin $(\mu \mathrm{I} \mathbf{U} / \mathbf{m L})^{+}$ & $21.4 \pm 22.0$ & $27.2 \pm 36.8$ & $33.8 \pm 33.3$ & $28.7 \pm 35.7$ & $22.6 \pm 20.4$ & $40.8 \pm 57.3$ & $11.9 \pm 7.0$ & $39.5 \pm 55.2$ \\
\hline HOMA-IR $^{+\Delta}$ & $5.45 \pm 5.79$ & $6.59 \pm 9.00$ & $5.35 \pm 3.21$ & $6.34 \pm 8.13$ & $5.13 \pm 4.27$ & $10.38 \pm 13.9$ & $2.92 \pm 1.96$ & $10.05 \pm 13.4$ \\
\hline $\begin{array}{l}\text { Triglycerides } \\
(\mathrm{mg} / \mathrm{dL})^{\&}\end{array}$ & $171 \pm 103$ & $130 \pm 40$ & $151 \pm 42$ & $135 \pm 41$ & $155 \pm 85$ & $142 \pm 52$ & $149 \pm 58$ & $144 \pm 52$ \\
\hline $\begin{array}{l}\text { Total cholesterol } \\
(\mathrm{mg} / \mathrm{dL})\end{array}$ & $194 \pm 26$ & $194 \pm 38$ & $178 \pm 23$ & $191 \pm 36$ & $191 \pm 33$ & $193 \pm 24$ & $219 \pm 21$ & $199 \pm 25$ \\
\hline $\operatorname{HDLc}(\mathrm{mg} / \mathrm{dL})$ & $36 \pm 10.3$ & $38 \pm 16.9$ & $43 \pm 18.8$ & $39 \pm 17.2$ & $36 \pm 14.8$ & $40 \pm 10.9$ & $48 \pm 13.4$ & $42 \pm 11.6$ \\
\hline $\operatorname{LDLc}(\mathrm{mg} / \mathrm{dL})^{\infty}$ & $120 \pm 27.4$ & $118 \pm 25.1$ & $99 \pm 26.4$ & $114 \pm 26.3$ & $114 \pm 26.7$ & $121 \pm 21.4$ & $150 \pm 27.7$ & $128 \pm 25.4$ \\
\hline VLDLc (mg/dL) & $33.9 \pm 20.7$ & $26.2 \pm 8.2$ & $30.3 \pm 8.4$ & $27.2 \pm 8.3$ & $30.9 \pm 17.2$ & $28.5 \pm 10.5$ & $30.0 \pm 11.8$ & $28.8 \pm 10.5$ \\
\hline $\begin{array}{l}\text { Apolipoprotein A1 } \\
(\mathrm{mg} / \mathrm{dL})^{\circ \&}\end{array}$ & $106 \pm 16.9$ & $123 \pm 28.7$ & $148 \pm 37.9$ & $128 \pm 31.5$ & $116 \pm 29.3$ & $124 \pm 12.4$ & $112 \pm 17.9$ & $119 \pm 15.0$ \\
\hline $\begin{array}{l}\text { Apolipoprotein B } \\
(\mathrm{mg} / \mathrm{dL})^{*}\end{array}$ & $121 \pm 25.8$ & $115 \pm 16.8$ & $80 \pm 46.8$ & $108 \pm 28.3$ & $116 \pm 20.9$ & $87 \pm 54.7$ & $134 \pm 37.5$ & $107 \pm 50.8$ \\
\hline $\begin{array}{l}\text { Total sAdiponectin } \\
(\mathrm{ng} / \mathrm{mL})^{+}\end{array}$ & $4421 \pm 3452$ & $3897 \pm 1769$ & $5946 \pm 5929$ & $4553 \pm 3646$ & $4598 \pm 3779$ & $3596 \pm 2364$ & $4861 \pm 1613$ & $3210 \pm 1466$ \\
\hline sResistin (ng/mL) & $10.3 \pm 4.1$ & $9.3 \pm 2.0$ & $14.3 \pm 2.3$ & $10.8 \pm 3.1$ & $10.5 \pm 3.3$ & $8.0 \pm 3.7$ & $11.7 \pm 6.7$ & $10.8 \pm 5.7$ \\
\hline sLeptin (ng/mL) & $55.8 \pm 31.1$ & $54.8 \pm 26.4$ & $72.6 \pm 41.1$ & $58.9 \pm 30.7$ & $55.4 \pm 30.9$ & $69.5 \pm 30.7$ & $57.4 \pm 26.7$ & $66.1 \pm 29.1$ \\
\hline
\end{tabular}

of conicity index in carriers of genotypes 12Pro/Pro and 12Pro/Ala was observed, along with increase in the HOMA-IR index magnitude when compared to 12Pro/Pro subjects (Figure 2(e)).

(iii) Decrease in resistin and leptin levels in individuals Gly- when compared to Gly+ phenotypes (Figure 2(d)); whereas subjects with PPARG2's Alaphenotype showed a decrease in HOMA-IR index, glucose, and insulin's basal levels and an increase in total adiponectin serum levels versus carriers of the Ala+ phenotype (Figure 2(f)).

\section{Discussion}

Based on the fact that gene polymorphisms related to energetic expenditure and adipogenesis can be associated with obesogenic phenotypes in different populations, this study focused on the possible relationship between functional polymorphisms Gly482Ser in PPARGC1A and Pro12Ala in PPARG2 with the accumulation and distribution of body fat and metabolic profile.
In the subjects of this study the mean in the BMI was of $26.8 \mathrm{~kg} / \mathrm{m}^{2}$, which was similar to some populations [3437] but greater than others [38-41]. In addition, we also noted in our cohort that BMI coexists with a fat mass percentage of 30.2 [37]. A recent report indicated that the distribution and quantity of adipose tissue and BMI are independent [42], moreover, in the present study we observed that accumulation and distribution of body fat tissue, levels of metabolic markers, soluble resistin and leptin, were higher when BMI increased, whereas levels of adiponectin and apolipoprotein A-1 decreased. Besides, we found increased metabolic markers when compared to other populations [3438].

These findings support the previously stated hypothesis that a variation on BMI and the accumulation of body fat on individuals have a genetic factor involved. In addition, these data suggest that genetic factors might be involved in triggering altered metabolic profile in our population. This finding is consistent with Chandalia's proposal that even if two ethnic groups present the same adipose tissue proportion, histological and functional differences can be found as manifestations of diverse chronic comorbidities [42]. 


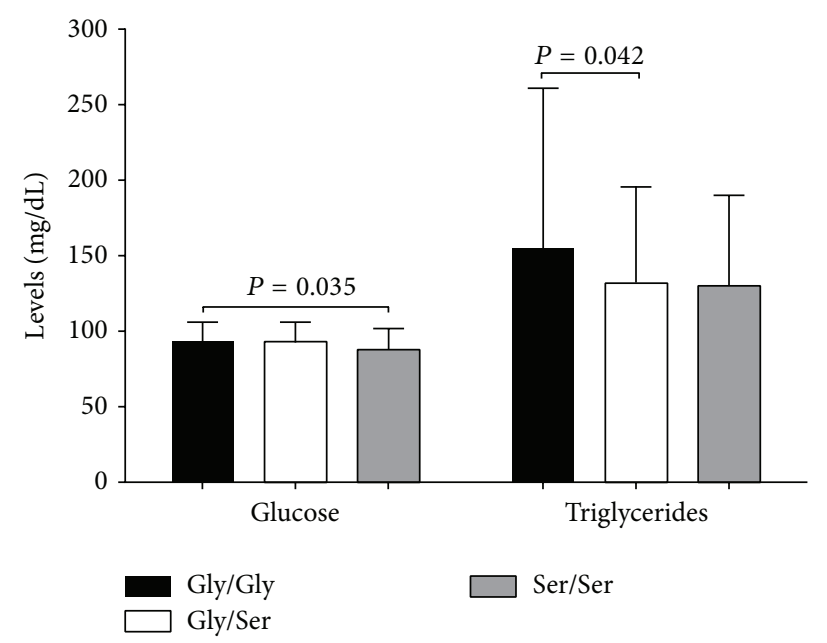

(a)

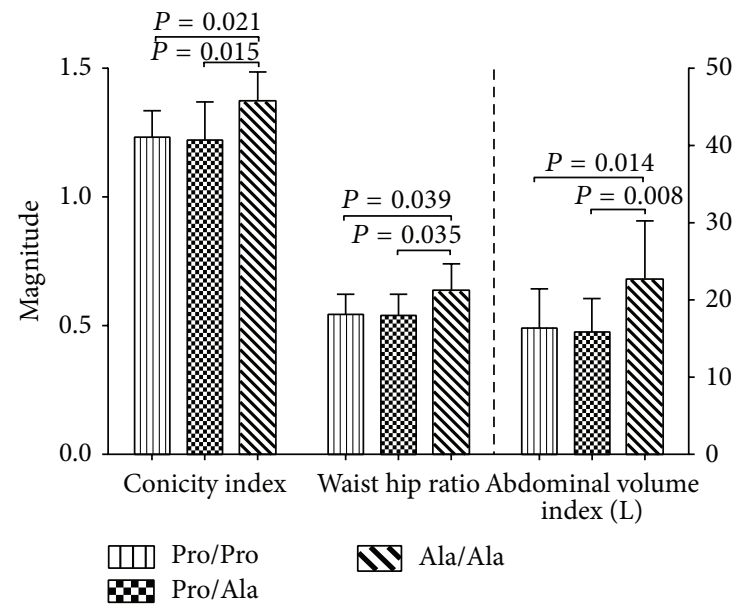

(c)

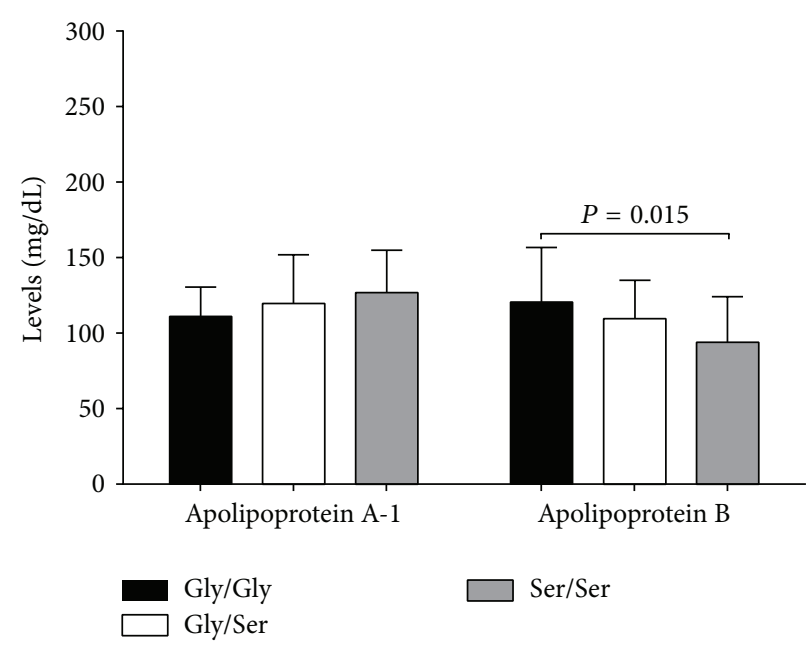

(b)

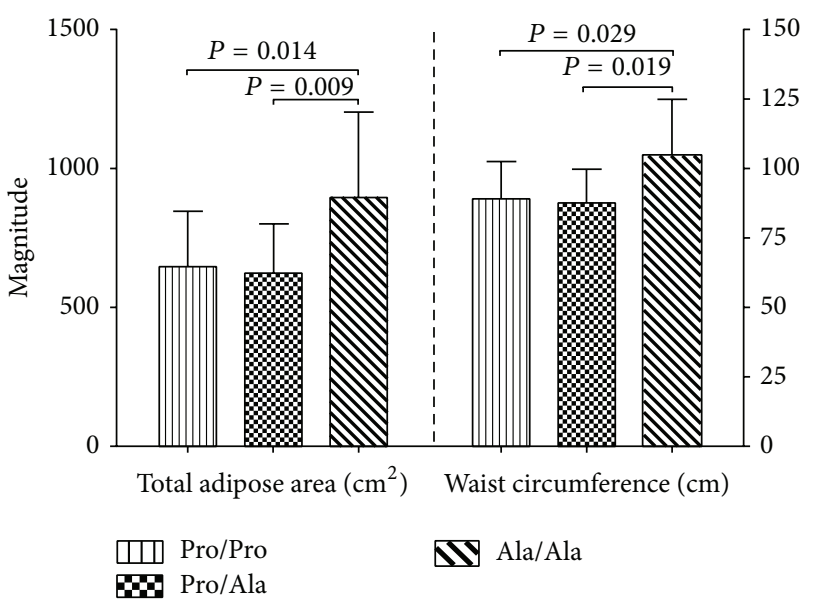

(d)

FIGURE 1: Metabolic markers, body dimensions and distribution of body fat storage measurements in study group by genotypes. (a) And (b) PPARGC1A Gly482Ser polymorphism; (c) and (d) Pro12Ala in PPARG2. One-way ANOVA test with Tukey's post hoc test. Data are means \pm SD. $n=375$.

In this regard, different studies performed since the 80's consistently reported a relationship between the increase of adipose tissue and metabolic disorders [43]. In our study, we determined the correlation of metabolic markers levels with adiposity indexes, to evaluate the metabolic imbalance caused by adipose tissue. We found a positive correlation of glucose, insulin, HOMA-IR and lipid profile with adiposity indexes, whereas HDLc and apolipoprotein A-1 levels showed a negative correlation.

These data are consistent with a meta-analysis previously published by Fulop et al., who stated that through the increase in adipose tissue, the insulin resistance and cardiovascular risk also increased [44]. It is also consistent with diverse studies that have demonstrated the importance of adipose tissue as a metabolic regulator based upon its secreting capacity $[45,46]$ while changes in the amount and distribution of adipose tissue may favor the development of secondary chronic illness $[44,47]$.
With regard to susceptibility genes involved in adipose tissue differentiation and regulation, the polymorphism Prol2Ala in PPARG2 was first described in 1997 by Chung-Jen et al. [48]. Further studies reported controversial results; nevertheless, most of them are consistent with the fact that allele 12Ala is associated with a greater risk of developing T2DM and gaining body weight on long term [49-51].

In our study, the allele 12Ala was observed to have a higher frequency when compared to populations from Palestine [52], Japan [19, 53], Turkey [54] and China [55]. This polymorphism was analyzed in different groups of Mexican people and the distribution found among them was similar to the present study $[48,56-59]$.

The supplementary analysis where we compare the carriers of different genotypes in PPARG2 polymorphism, according to their classification by BMI, in groups with normal range and pre-obesity, showed no difference, except for HDLc 


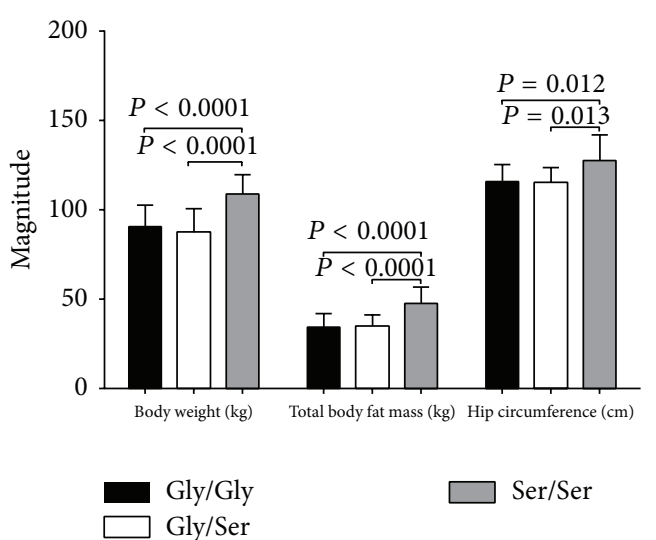

(a)

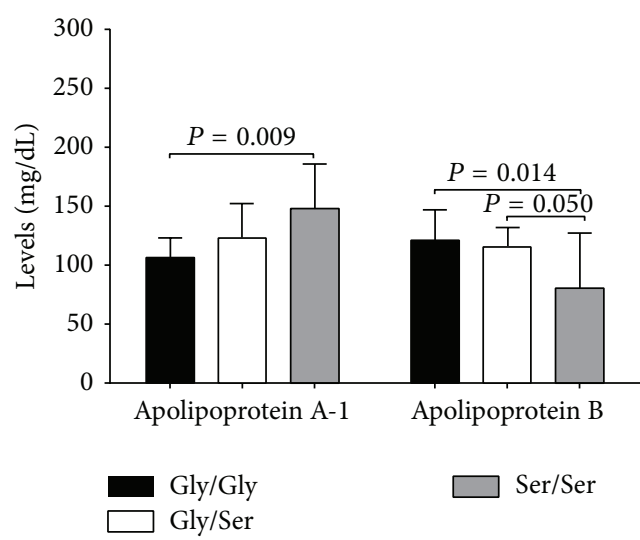

(c)

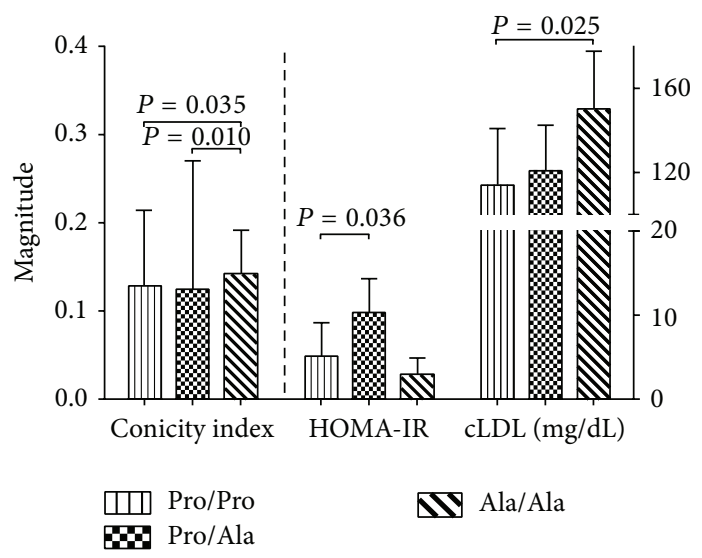

(e)

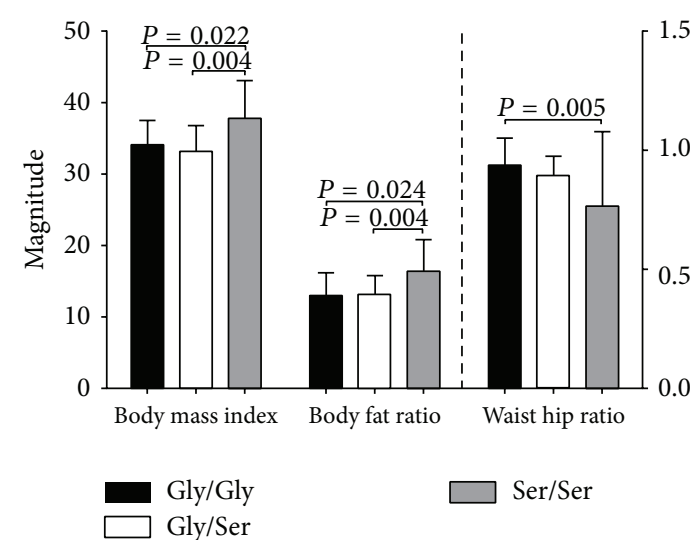

(b)

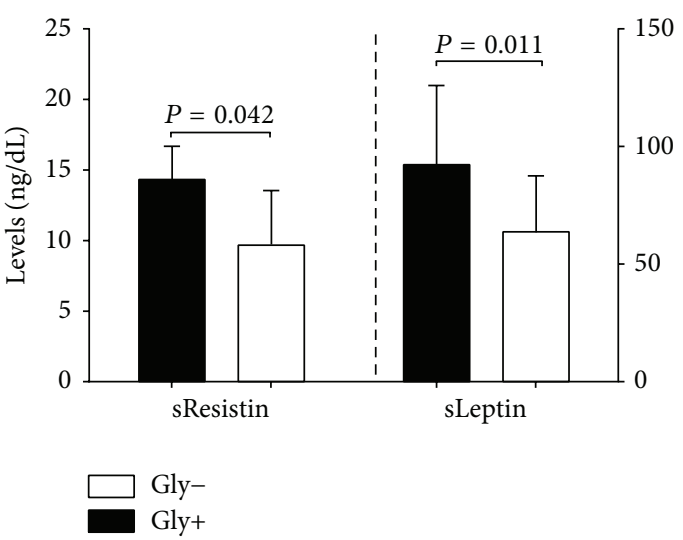

(d)

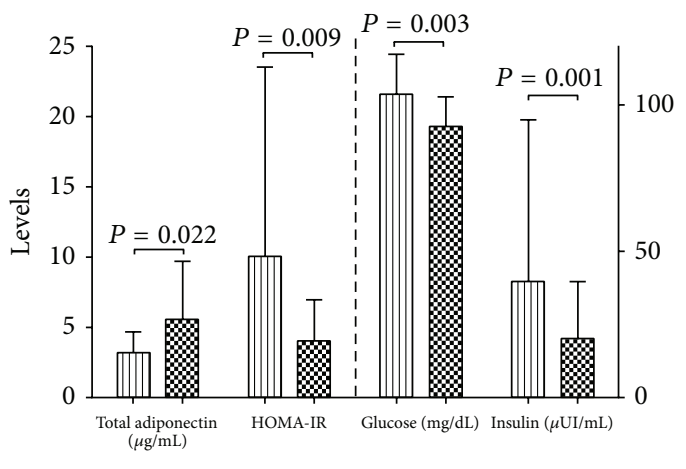

미 Ala+

W Ala-

(f)

Figure 2: Metabolic markers, adipokines, and body dimensions and distribution of body fat storage measurements in obese subjects by genotypes and phenotypes. (a), (b), (c) and (d) PPARGC1A Gly482Ser polymorphism; (e) and (f) Prol2Ala in PPARG2. One way ANOVA test with Tukey's post hoc test, and *Student's $t$-test. Polymorphism PPARGC1A: phenotype Gly- (Genotype 482Ser/Ser) and, phenotype Gly+ (genotypes 482Gly/Ser plus 482Gly/Gly). Polymorphism PPARG2: phenotype Ala+ (genotypes 12Pro/Ala plus 12Ala/Ala) and phenotype Ala- (genotype 12Pro/Pro). Data are means \pm SD. $n=78$.

levels in subjects with preobesity, while in the carriers of the phenotype Ala+ in obese group, an increase in metabolic markers was observed.

It is important to highlight that we are the first group reporting that $12 \mathrm{Ala} / \mathrm{Ala}$ genotype carriers show increase in adiposity indexes, such as conicity and abdominal volume indexes and total adipose area, which are indicative of abdominal obesity, along with a difference in the distribution of Pro12Ala in PPARG2 polymorphism among subjects with obesity when compared to nonobese subjects. 
In this sense, such differences can be explained by the fact that PPARG2 polymorphism increases its gene expression on abdominal adipose tissue, which in turn boost adipocyte differentiation and maturation in that body region and finally generates a metabolic pathogenic process [60-62]. The relevance of such finding lies in the existence of a potential pathogenic capacity of abdominal obesity that has led to consider it an important risk factor, both by itself and as a promoter of diseases that can generate comorbidity such as T2DM and cardiovascular disease.

On the other hand, abdominal adipose tissue is the main producer of the proinflammatory adipokines [63]. We found lower levels of total adiponectin, higher HOMA-IR and glucose in obese individuals carrying phenotype Ala+ than Ala- phenotype of PPARG2 gene. Such results have not been reported previously and more studies are required to look for a possible explanation. However, we hypothesize that it could be related to PPARG2's transrepressing activity [64, 65].

The variations found in our results, cannot be exclusive for PPARG2 and can be influenced by other protein interactions. Among the possible interacting molecules, PGC- $1 \alpha$ stands out and has been proposed as an important orchestrator in glucose metabolism. The Gly482Ser PPARGC1A polymorphism has been associated with T2DM, hypertension, and Parkinson's disease [66-70].

Although the distribution of Gly482Ser in PPARGC1A showed no difference in our cohort, we found a heterozygous frequency of $39 \%$ and $38 \%$, in normal range and preobese groups, respectively. This frequency is comparable with reports from other populations, while a $30 \%$ frequency was found for allele 482Ser, similar to others [66-68]. Previous studies report an association between allele 482Ser and the presence of T2DM in populations from China [71], Japan [72], Caucasians [73], and Korean [70] as well as a link with hypertension in German common people [11] and early insulin secretion in Pima Indians [12]. However, in our population there are no reports in this regard.

A relevant finding in our study was the presence of a greater magnitude in obesity indexes (e.g., body weight, BMI, body fat ratio, and total body fat mass) are present in carriers of genotype $482 \mathrm{Ser} / \mathrm{Ser}$. This could be explained by the fact that PGC- $1 \alpha$ plays a role in adipocyte differentiation [74], which rises in subjects with adipose tissue hyperplasic and hypertrophic, individuals that can be catalogued as obese (i.e., $\mathrm{BMI}>30 \mathrm{~kg} / \mathrm{m}^{2}$ ).

Additionally, the involvement of PPARGC1A polymorphism in metabolic imbalance was evaluated. In this context, we found that allele 482 Ser was associated with diminished glucose, triglycerides and apolipoprotein B (on heterozygote or homozygote polymorphic genotypes carriers) whilst, apolipoprotein A-1 levels was increased, at the same time, resistin and leptin levels were low on Gly- phenotype carriers. Nevertheless, no previous reports exist; hence, there is no referral for comparison. We suggest that carriers of allele 482Ser have a healthy metabolic profile.

In summary, we observed a heterogeneous profile in the polymorphic genotypes and phenotypes for both genes: 12Ala/Ala polymorphic genotype in PPARG2, an association with increasing magnitude of indicators of preferential accumulation of abdominal fat was found, whereas for polymorphic genotype 482Ser/Ser in PPARGC1A the connection is represented by a decreased in metabolic markers and adipokine levels. Unfortunately we were not able to show individuals carrying the polymorphic genotypes in both genes in this report. This implies we could not know the phenotype behavior.

These leads to the proposal of future investigations regarding these molecules since they could be taken into account as therapeutic targets and/or as surrogate markers of individuals that have an increased risk comorbidity development. This could lead to novel clinical and pharmacological approaches in glucose and adipose metabolism control.

\section{Conclusions}

In obese Mexican-Mestizos, the combination of alleles $482 \mathrm{Ser}$ in PPARGC1A and 12Pro in PPARG2 presents a reduced metabolic risk profile; even adiposity indexes are increased.

\section{Conflict of Interests}

The authors declare that there is no conflict of interests regarding the publication of this paper.

\section{Authors' Contribution}

Mónica Vázquez-Del Mercado and Milton-Omar GuzmánOrnelas equally contributed to this work.

\section{Acknowledgments}

Our work is supported by grant from COECYTJAL-UDG 778 and grant from PRO-SNI/UDG 2008-2009 to Rosa-Elena Navarro-Hernandez.

\section{References}

[1] J. A. J. Martyn, M. Kaneki, and S. Yasuhara, "Obesity-induced insulin resistance and hyperglycemia: etiologic factors and molecular mechanisms," Anesthesiology, vol. 109, no. 1, pp. 137148, 2008.

[2] M. M. J. van Greevenbroek, C. G. Schalkwijk, and C. D. A. Stehouwer, "Obesity-associated low-grade inflammation in type 2 diabetes mellitus: causes and consequences," Netherlands Journal of Medicine, vol. 71, no. 4, pp. 174-187, 2013.

[3] Q. Xia and S. F. A. Grant, "The genetics of human obesity," Annals of the New York Academy of Sciences, vol. 1281, no. 1, pp. 178-190, 2013.

[4] W. K. Chung and R. L. Leibel, "Considerations regarding the genetics of obesity," Obesity, vol. 16, no. 3, pp. S33-S39, 2008.

[5] J. Naukkarinen, A. Rissanen, J. Kaprio, and K. H. Pietiläinen, "Causes and consequences of obesity: the contribution of recent twin studies," International Journal of Obesity, vol. 36, no. 8, pp. 1017-1024, 2012.

[6] I. Dahlman and P. Arner, "Genetics of adipose tissue biology," Progress in Molecular Biology and Translational Science, vol. 94, pp. 39-74, 2010. 
[7] H. Esterbauer, H. Oberkofler, F. Krempler, and W. Patsch, "Human peroxisome proliferator activated receptor gamma coactivator 1 (PPARGC1) gene: cDNA sequence, genomic organization, chromosomal localization, and tissue expression," Genomics, vol. 62, no. 1, pp. 98-102, 1999.

[8] J. Ek, G. Andersen, S. A. Urhammer et al., "Mutation analysis of peroxisome proliferator-activated receptor-gamma coactivator1 (PGC-1) and relationships of identified amino acid polymorphisms to Type II diabetes mellitus," Diabetologia, vol. 44, no. 12, pp. 2220-2226, 2001.

[9] S.-L. Zhang, W.-S. Lu, L. Yan et al., "Association between peroxisome proliferator-activated receptor- $\gamma$ coactivator- $1 \alpha$ gene polymorphisms and type 2 diabetes in southern Chinese population: role of altered interaction with myocyte enhancer factor 2C," Chinese Medical Journal, vol. 120, no. 21, pp. 1878-1885, 2007.

[10] G. S. Sainani and R. A. Karatela, "Plasma leptin in insulinresistant and insulin-nonresistant coronary artery disease and its association with cardio-metabolic risk factors among Asian Indians," Metabolic Syndrome and Related Disorders, vol. 7, no. 4, pp. 335-340, 2009.

[11] H. Oberkofler, B. Hölzl, H. Esterbauer et al., "Peroxisome proliferator-activated receptor- $\gamma$ coactivator-1 gene locus: associations with hypertension in middle-aged men," Hypertension, vol. 41, no. 2, pp. 368-372, 2003.

[12] Y. L. Muller, C. Bogardus, O. Pedersen, and L. Baier, "A Gly482Ser missense mutation in the peroxisome proliferatoractivated receptor $\gamma$ coactivator- 1 is associated with altered lipid oxidation and early insulin secretion in Pima Indians," Diabetes, vol. 52, no. 3, pp. 895-898, 2003.

[13] F. Francès, F. Verdú, O. Portolés et al., "PPAR- $\alpha$ L162V and PGC1 G482S gene polymorphisms, but not $P P A R-\gamma$ P12A, are associated with alcohol consumption in a Spanish Mediterranean population," Clinica Chimica Acta, vol. 398, no. 1-2, pp. 70-74, 2008.

[14] M. I. Lefterova, A. K. Haakonsson, M. A. Lazar, and S. Mandrup, "PPAR $\gamma$ and the global map of adipogenesis and beyond," Trends in Endocrinology \& Metabolism, vol. 25, no. 6, pp. 293302, 2014.

[15] M. Ahmadian, J. M. Suh, N. Hah et al., "PPARgamma signaling and metabolism: the good, the bad and the future," Nature Medicine, vol. 19, no. 5, pp. 557-566, 2013.

[16] Z. E. Floyd and J. M. Stephens, "Controlling a master switch of adipocyte development and insulin sensitivity: covalent modifications of PPAR $\gamma$," Biochimica et Biophysica Acta: Molecular Basis of Disease, vol. 1822, no. 7, pp. 1090-1095, 2012.

[17] S. Cinti, "Transdifferentiation properties of adipocytes in the adipose organ," American Journal of Physiology: Endocrinology and Metabolism, vol. 297, no. 5, pp. E977-E986, 2009.

[18] J. Yamamoto, S. Kageyama, M. Nemoto et al., "PPAR 22 Pro12Ala polymorphism and insulin resistance in Japanese hypertensive patients," Hypertension Research, vol. 25, no. 1, pp. 25-29, 2002.

[19] F. Wang, T. Tahara, T. Arisawa et al., "Polymorphism of peroxisome proliferator-activated receptor gamma is not associated to Japanese ulcerative colitis," Hepato-Gastroenterology, vol. 55, no. 81, pp. 73-75, 2008.

[20] M. Stumvoll and H. Häring, "The peroxisome proliferatoractivated receptor- $\gamma 2$ Pro12Ala polymorphism," Diabetes, vol. 51, no. 8, pp. 2341-2347, 2002.
[21] C. Gorodezky, C. Alaez, M. N. Vázquez-García et al., “The genetic structure of Mexican Mestizos of different locations: tracking back their origins through MHC genes, blood group systems, and microsatellites," Human Immunology, vol. 62, no. 9, pp. 979-991, 2001.

[22] World-Health-Organization, Obesity: Preventing and Managing the Global Epidemic, 1st edition, 2012.

[23] H. C. Lukaski, "Body mass index, bioelectrical impedance, and body composition," Nutrition, vol. 17, no. 1, pp. 55-56, 2001.

[24] H. C. Lukaski, "Regional bioelectrical impedance analysis: applications in health and medicine," Acta Diabetologica, vol. 40, no. 1, pp. S196-S199, 2003.

[25] R. Ness-Abramof and C. M. Apovian, "Waist circumference measurement in clinical practice," Nutrition in Clinical Practice, vol. 23, no. 4, pp. 397-404, 2008.

[26] D. N. Miller, J. E. Bryant, E. L. Madsen, and W. C. Ghiorse, "Evaluation and optimization of DNA extraction and purification procedures for soil and sediment samples," Applied and Environmental Microbiology, vol. 65, no. 11, pp. 4715-4724, 1999.

[27] M.-O. Guzman-Ornelas, E. Chavarria-Avila, J.-F. Munoz-Valle et al., "Association of ADIPOQ $+45 \mathrm{~T}>\mathrm{G}$ polymorphism with body fat mass and blood levels of soluble adiponectin and inflammation markers in a Mexican-Mestizo population," Diabetes, Metabolic Syndrome and Obesity: Targets and Therapy, vol. 5, pp. 369-378, 2012.

[28] F. Guerrero-Romero and M. Rodríguez-Morán, “Abdominal volume index. An anthropometry-based index for estimation of obesity is strongly related to impaired glucose tolerance and type 2 diabetes mellitus," Archives of Medical Research, vol. 34, no. 5, pp. 428-432, 2003.

[29] M. Garaulet, J. J. Hernández-Morante, F. J. Tébar, S. Zamora, and M. Canteras, "Two-dimensional predictive equation to classify visceral obesity in clinical practice," Obesity, vol. 14, no. 7, pp. 1181-1191, 2006.

[30] M. R. Mamtani and H. R. Kulkarni, "Predictive performance of anthropometric indexes of central obesity for the risk of type 2 diabetes," Archives of Medical Research, vol. 36, no. 5, pp. 581589, 2005.

[31] D. R. Matthews, J. P. Hosker, A. S. Rudenski, B. A. Naylor, D. F. Treacher, and R. C. Turner, "Homeostasis model assessment: insulin resistance and $\beta$-cell function from fasting plasma glucose and insulin concentrations in man," Diabetologia, vol. 28, no. 7, pp. 412-419, 1985.

[32] W. T. Friedewald, R. I. Levy, and D. S. Fredrickson, "Estimation of the concentration of low-density lipoprotein cholesterol in plasma, without use of the preparative ultracentrifuge.," Clinical Chemistry, vol. 18, no. 6, pp. 499-502, 1972.

[33] G. H. Hardy, "Mendelian proportions in a mixed population," Science, vol. 28, no. 706, pp. 49-50, 1908.

[34] E. P. de Oliveira, M. D. D. A. de Lima, and M. L. A. de Souza, "Metabolic syndrome, its phenotypes, and insulin resistance by HOMA-IR," Arquivos Brasileiros de Endocrinologia e Metabologia, vol. 51, no. 9, pp. 1506-1515, 2007.

[35] S. Benozzi, F. Ordonez, N. Polini, C. Alvarez, J. Sellest, and R. I. Coniglio, "Insulin-resistance and metabolic syndrome in patients with coronary heart disease defined by angiography," Medicina, vol. 69, no. 2, pp. 221-228, 2009.

[36] R. Dalan, M. Jong, S. P. Chan et al., "High-sensitivity C-reactive protein concentrations among patients with and without diabetes in a multiethnic population of Singapore: CREDENCE Study," Diabetes, Metabolic Syndrome and Obesity: Targets and Therapy, vol. 3, pp. 187-195, 2010. 
[37] S. H. Chu, M. K. Lee, K. Y. Ahn et al., "Chemerin and adiponectin contribute reciprocally to metabolic syndrome," PLoS ONE, vol. 7, no. 4, Article ID e34710, 2012.

[38] D. Tsuriya, H. Morita, T. Morioka et al., "Significant correlation between visceral adiposity and high-sensitivity C-reactive protein (hs-CRP) in Japanese subjects," Internal Medicine, vol. 50, no. 22, pp. 2767-2773, 2011.

[39] W. Ou, X. Liu, Y. Shen et al., "Association of CVD candidate gene polymorphisms with ischemic stroke and cerebral hemorrhage in chinese individuals," PLoS ONE, vol. 9, no. 8, Article ID e105516, 2014.

[40] D. Vasudevan, A. L. Stotts, S. Mandayam, and L. A. Omegie, "Comparison of BMI and anthropometric measures among South Asian Indians using standard and modified criteria," Public Health Nutrition, vol. 14, no. 5, pp. 809-816, 2010.

[41] J. Harbron, L. van der Merwe, M. G. Zaahl, M. J. Kotze, and M. Senekal, "Fat mass and obesity-associated (FTO) gene polymorphisms are associated with physical activity, food intake, eating behaviors, psychological health, and modeled change in body mass index in overweight/obese Caucasian adults," Nutrients, vol. 6, no. 8, pp. 3130-3152, 2014.

[42] M. Chandalla, P. Lin, T. Seenivasan et al., "Insulin resistance and body fat distribution in South Asian men compared to Caucasian men," PLoS ONE, vol. 2, no. 8, article e812, 2007.

[43] N. M. Kaplan, "The deadly quartet. Upper-body obesity, glucose intolerance, hypertriglyceridemia, and hypertension," Archives of Internal Medicine, vol. 149, no. 7, pp. 1514-1520, 1989.

[44] T. Fulop, D. Tessier, and A. Carpentier, "The metabolic syndrome," Pathologie Biologie, vol. 54, no. 7, pp. 375-386, 2006.

[45] K. Karastergiou and V. Mohamed-Ali, "The autocrine and paracrine roles of adipokines," Molecular and Cellular Endocrinology, vol. 318, no. 1-2, pp. 69-78, 2010.

[46] S. Galic, J. S. Oakhill, and G. R. Steinberg, "Adipose tissue as an endocrine organ," Molecular and Cellular Endocrinology, vol. 316, no. 2, pp. 129-139, 2010.

[47] C. de Luca and J. M. Olefsky, "Inflammation and insulin resistance," FEBS Letters, vol. 582, no. 1, pp. 97-105, 2008.

[48] Y. Chung-Jen, B. A. Beamer, C. Negri et al., "Molecular scanning of the human peroxisome proliferator activated receptor $\gamma$ $(\mathrm{hPPAR} \gamma)$ gene in diabetic Caucasians: identification of a Prol2Ala PPAR $\gamma 2$ missense mutation," Biochemical and Biophysical Research Communications, vol. 241, no. 2, pp. 270-274, 1997.

[49] S. S. Deeb, L. Fajas, M. Nemoto et al., "A Pro12Ala substitution in PPAR $\gamma 2$ associated with decreased receptor activity, lower body mass index and improved insulin sensitivity," Nature Genetics, vol. 20, no. 3, pp. 284-287, 1998.

[50] J. Kuliczkowska, A. Filus, A. Trzmiel et al., "PPAR- $\gamma 2$ Pro12Ala polymorphism in the population of obese and non-obese men of the city of Wroclaw," Endokrynologia Polska, vol. 59, no. 4, pp. 312-315, 2008.

[51] N. A. West, M. N. Haan, and H. Morgenstern, "The PPARgamma Pro12Ala polymorphism and risk of cognitive impairment in a longitudinal study," Neurobiology of Aging, vol. 31, no. 5, pp. 741-746, 2010.

[52] S. Ereqat, A. Nasereddin, K. Azmi, Z. Abdeen, and R. Amin, "Impact of the pro12Ala polymorphism of the PPAR-gamma 2 gene on metabolic and clinical characteristics in the palestinian type 2 diabetic patients," PPAR Research, vol. 2009, Article ID 874126, 5 pages, 2009.
[53] Y. Aoyagi, S. Nagata, T. Kudo et al., "Peroxisome proliferatoractivated receptor $\gamma 2$ mutation may cause a subset of ulcerative colitis," Pediatrics International, vol. 52, no. 5, pp. 729-734, 2010.

[54] O. Atug, V. Tahan, F. Eren et al., "Prol2Ala polymorphism in the peroxisome proliferator-activated receptor-gamma (PPAR $\gamma$ ) gene in inflammatory bowel disease," Journal of Gastrointestinal and Liver Diseases, vol. 17, no. 4, pp. 433-437, 2008.

[55] U. K. Shrestha, O. Karimi, J. B. A. Crusius et al., "Distribution of peroxisome proliferator-activated receptor-gamma polymorphisms in Chinese and Dutch patients with inflammatory bowel disease," Inflammatory Bowel Diseases, vol. 16, no. 2, pp. 312-319, 2010.

[56] J. Duran-Gonzalez, I. Ortiz, E. Gonzales et al., "Association study of candidate gene polymorphisms and obesity in a young Mexican-American population from South Texas," Archives of Medical Research, vol. 42, no. 6, pp. 523-531, 2011.

[57] B. I. Estrada-Velasco, M. Cruz, V. Madrid-Marina, G. A. Martínez-Nava, J. Gomez-Zamudio, and A. I. Burguete-García, "IRS1, TCF7L2, ADRB1, PPARG, and HHEX polymorphisms associated with atherogenic risk in Mexican population," BioMed Research International, vol. 2013, Article ID 394523, 7 pages, 2013.

[58] M. A. Gamboa-Meléndez, A. Huerta-Chagoya, H. MorenoMacías et al., "Contribution of common genetic variation to the risk of type 2 diabetes in the Mexican Mestizo population," Diabetes, vol. 61, no. 12, pp. 3314-3321, 2012.

[59] L. E. Martínez-Gómez, M. Cruz, G. A. Martínez-Nava et al., "A replication study of the IRS1, CAPN10, TCF7L2, and PPARG gene polymorphisms associated with type 2 diabetes in two different populations of Mexico," Annals of Human Genetics, vol. 75, no. 5, pp. 612-620, 2011.

[60] J. Berger and D. E. Moller, "The mechanisms of action of PPARs," Annual Review of Medicine, vol. 53, pp. 409-435, 2002.

[61] J. P. Berger, T. E. Akiyama, and P. T. Meinke, "ARsARtherapeutic targets for metabolic disease," Trends in Pharmacological Sciences, vol. 26, no. 5, pp. 244-251, 2005.

[62] B. Fève, "Adipogenesis: cellular and molecular aspects," Best Practice \& Research Clinical Endocrinology \& Metabolism, vol. 19, no. 4, pp. 483-499, 2005.

[63] M. Guerro-Millo, "Adipose tissue and adipokines: for better or worse," Diabetes \& Metabolism, vol. 30, no. 1, pp. 13-19, 2004.

[64] M. Mansour, "The roles of peroxisome proliferator-activated receptors in the metabolic syndrome," in Progress in Molecular Biology and Translational Science, vol. 121, chapter 7, pp. 217266, Elsevier, 2014.

[65] F. A. Monsalve, R. D. Pyarasani, F. Delgado-Lopez, and R. Moore-Carrasco, "Peroxisome proliferator-activated receptor targets for the treatment of metabolic diseases," Mediators of Inflammation, vol. 2013, Article ID 549627, 18 pages, 2013.

[66] A. Bhat, A. Koul, E. Rai, S. Sharma, and M. K. Dhar, "PGC-1 $\alpha$ Thr394Thr and Gly482Ser variants are significantly associated with T2DM in two North Indian populations: a replicate casecontrol study," Human Genetics, vol. 121, no. 5, pp. 609-614, 2007.

[67] J. Clark, S. Reddy, K. Zheng, R. A. Betensky, and D. K. Simon, "Association of PGC-1alpha polymorphisms with age of onset and risk of Parkinson's disease," BMC Medical Genetics, vol. 12, article 69, 2011.

[68] V. Ginevičiene, V. Pranculis, V. Jakaitiene, V. Milašius, and V. Kučinskas, "Genetic variation of the human ACE and ACTN3 genes and their association with functional muscle properties in 
Lithuanian elite athletes," Medicina, vol. 47, no. 5, pp. 284-290, 2011.

[69] E. Ingelsson, L. Bennet, M. Ridderstråle, M. Söderström, L. Råstam, and U. Lindblad, "The PPARGC1A Gly482Ser polymorphism is associated with left ventricular diastolic dysfunction in men," BMC Cardiovascular Disorders, vol. 8, article 37, 2008.

[70] J. H. Kim, H. D. Shin, B. L. Park et al., "Peroxisome proliferatoractivated receptor gamma coactivator 1 alpha promoter polymorphisms are associated with early-onset type 2 diabetes mellitus in the Korean population," Diabetologia, vol. 48, no. 7 , pp. 1323-1330, 2005.

[71] M.-C. Hsieh, K.-D. Lin, K.-J. Tien et al., "Common polymorphisms of the peroxisome proliferator-activated receptor$\gamma$ (Pro12Ala) and peroxisome proliferator-activated receptor$\gamma$ coactivator-1 (Gly482Ser) and the response to pioglitazone in Chinese patients with type 2 diabetes mellitus," Metabolism: Clinical and Experimental, vol. 59, no. 8, pp. 1139-1144, 2010.

[72] Y. Okauchi, H. Iwahashi, K. Okita et al., "PGC-1 $\alpha$ Gly482Ser polymorphism is associated with the plasma adiponectin level in type 2 diabetic men," Endocrine Journal, vol. 55, no. 6, pp. 991-997, 2008.

[73] L. Andrulionytè, J. Zacharova, J.-L. Chiasson, and M. Laakso, "Common polymorphisms of the PPAR- $\gamma 2$ (Pro12Ala) and $P G C-1 \alpha$ (Gly482Ser) genes are associated with the conversion from impaired glucose tolerance to type 2 diabetes in the STOPNIDDM trial," Diabetologia, vol. 47, no. 12, pp. 2176-2184, 2004.

[74] H. Liang and W. F. Ward, "PGC-1 $\alpha$ : a key regulator of energy metabolism," American Journal of Physiology: Advances in Physiology Education, vol. 30, no. 4, pp. 145-151, 2006. 

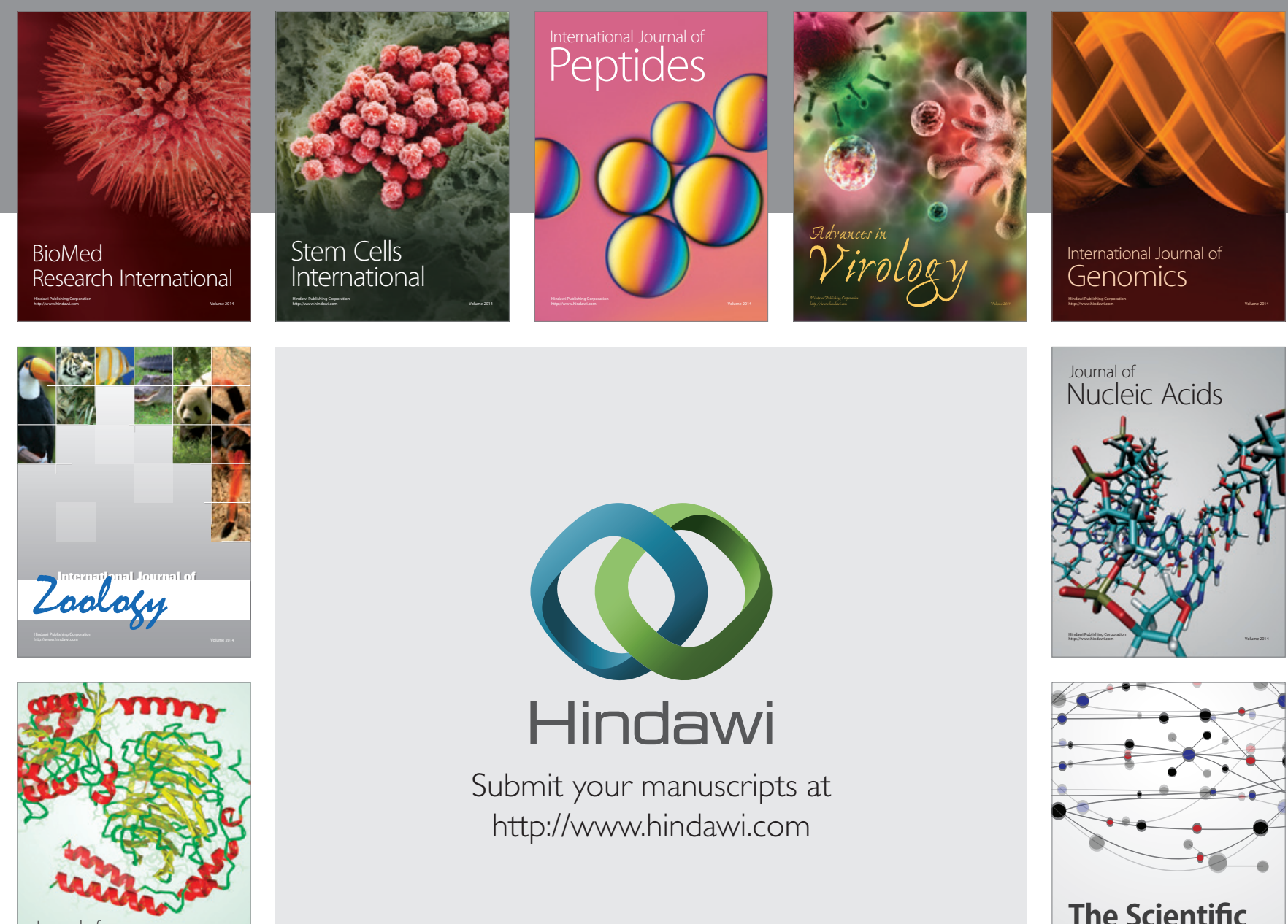

Submit your manuscripts at

http://www.hindawi.com

Journal of
Signal Transduction
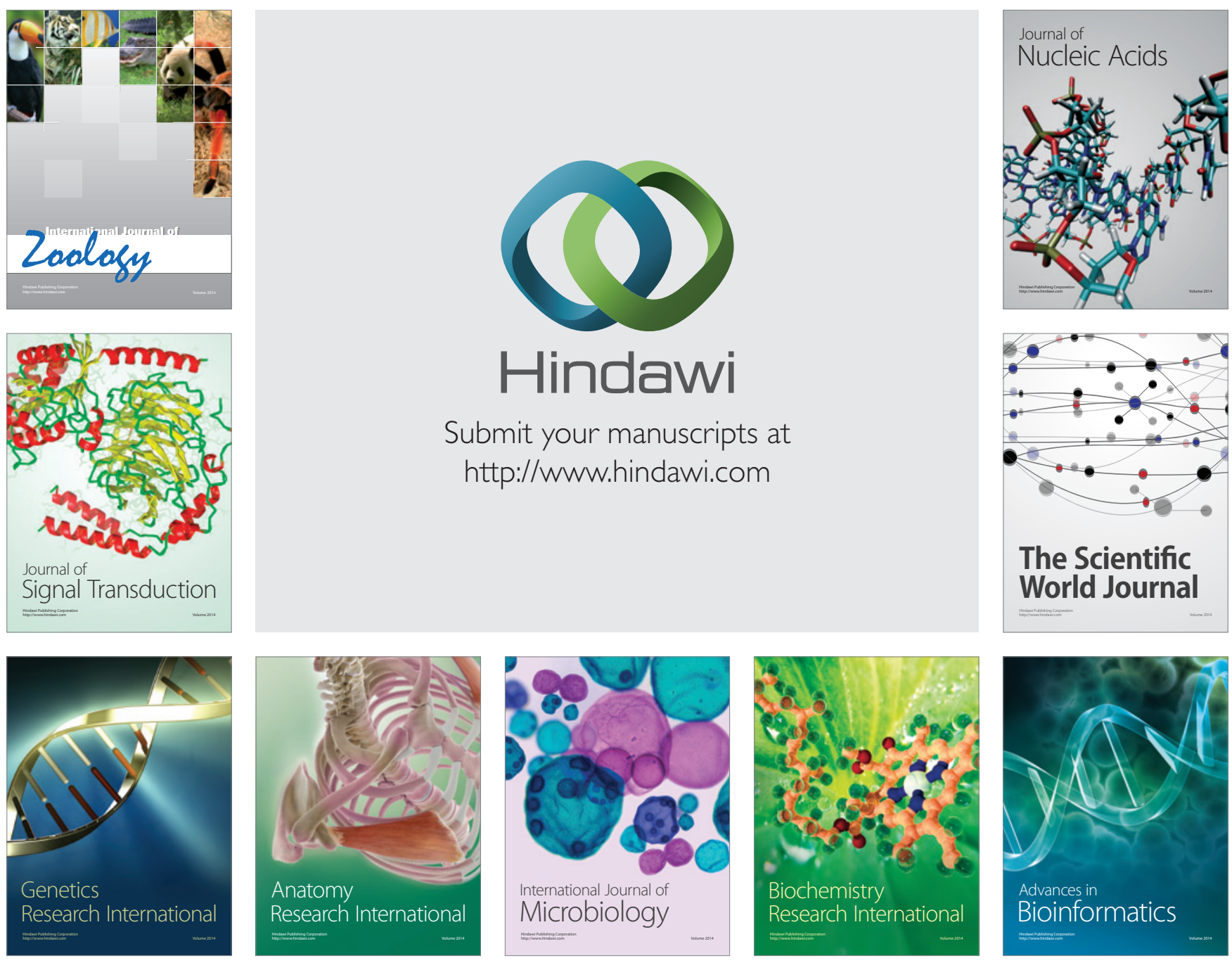

The Scientific World Journal
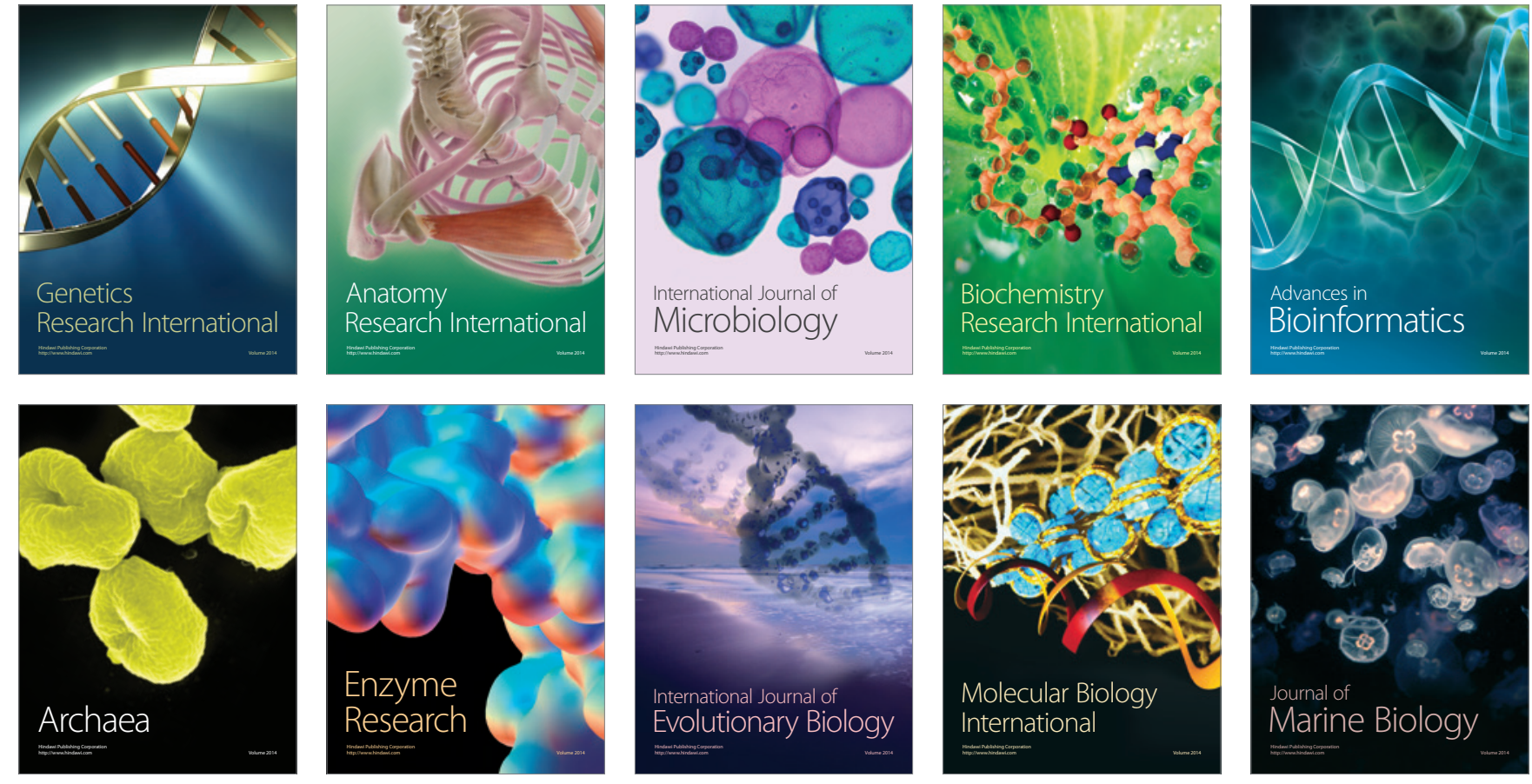